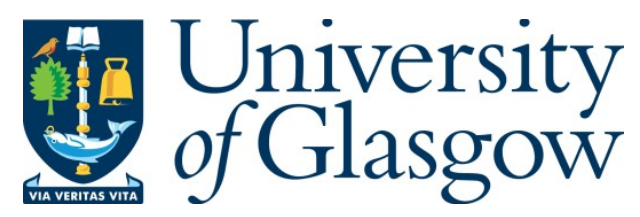

Fracchia, G., Biggs, J. D. and Ceriotti, M. (2021) Analytical low-jerk reorientation maneuvers for multi-body spacecraft structures. Acta Astronautica, 178, pp. 1-14.

(doi: 10.1016/j.actaastro.2020.08.020)

This is the Author Accepted Manuscript.

There may be differences between this version and the published version. You are advised to consult the publisher's version if you wish to cite from it.

https://eprints.gla.ac.uk/222685/

Deposited on: 28 August 2020

Enlighten - Research publications by members of the University of Glasgow http://eprints.gla.ac.uk 


\title{
Analytical Low-Jerk Reorientation Maneuvers for Multi-Body Spacecraft Structures
}

\author{
Guido Fracchia $^{\mathrm{a}, 1, *}$, James D. Biggs ${ }^{\mathrm{a}, 2}$, Matteo Ceriotti ${ }^{\mathrm{b}, 3}$ \\ ${ }^{a}$ Politecnico di Milano, 20156 Milano, Italy \\ ${ }^{b}$ University of Glasgow, Glasgow, G12 8QQ, UK
}

\begin{abstract}
A low-jerk attitude guidance method is developed, based on an analytical smoothing of a bang-off-bang maneuver. A set of closed-form equations are derived and used to plan constrained low-jerk maneuvers, with prescribed boundary conditions, inertia, time and maximum control torque. The guidance law is first developed for one-dimensional and then three-dimensional rotations using two different approaches: (i) by designing a rotation about the Euler axis and (ii) by using the inverse kinematics equations. A generic model of the torque induced by a multi-body appendage is derived using the lumpedparameter method. This method can also be used to approximate the dynamic behavior of flexible appendages. The simulations results show that the smoothing techniques reduce the excitation of multi-body and flexible structures during a slew maneuver.
\end{abstract}

Keywords: attitude control, multi-body spacecraft, flexible spacecraft, rest-to-rest maneuver, angular jerk

\section{Nomenclature}

ARF appendage reference frame

BRF body reference frame

\footnotetext{
${ }^{*}$ Corresponding author

Email addresses: guido.fracchia@mail.polimi.it (Guido Fracchia), jamesdouglas.biggs@polimi.it (James D. Biggs), Matteo.Ceriotti@glasgow.ac.uk (Matteo Ceriotti) ${ }^{1} \mathrm{MSc}$ Graduate in Space Engineering.

${ }^{2}$ Associate Professor, Department of Aerospace Science and Technology.

${ }^{3}$ Lecturer, James Watt School of Engineering.
} 


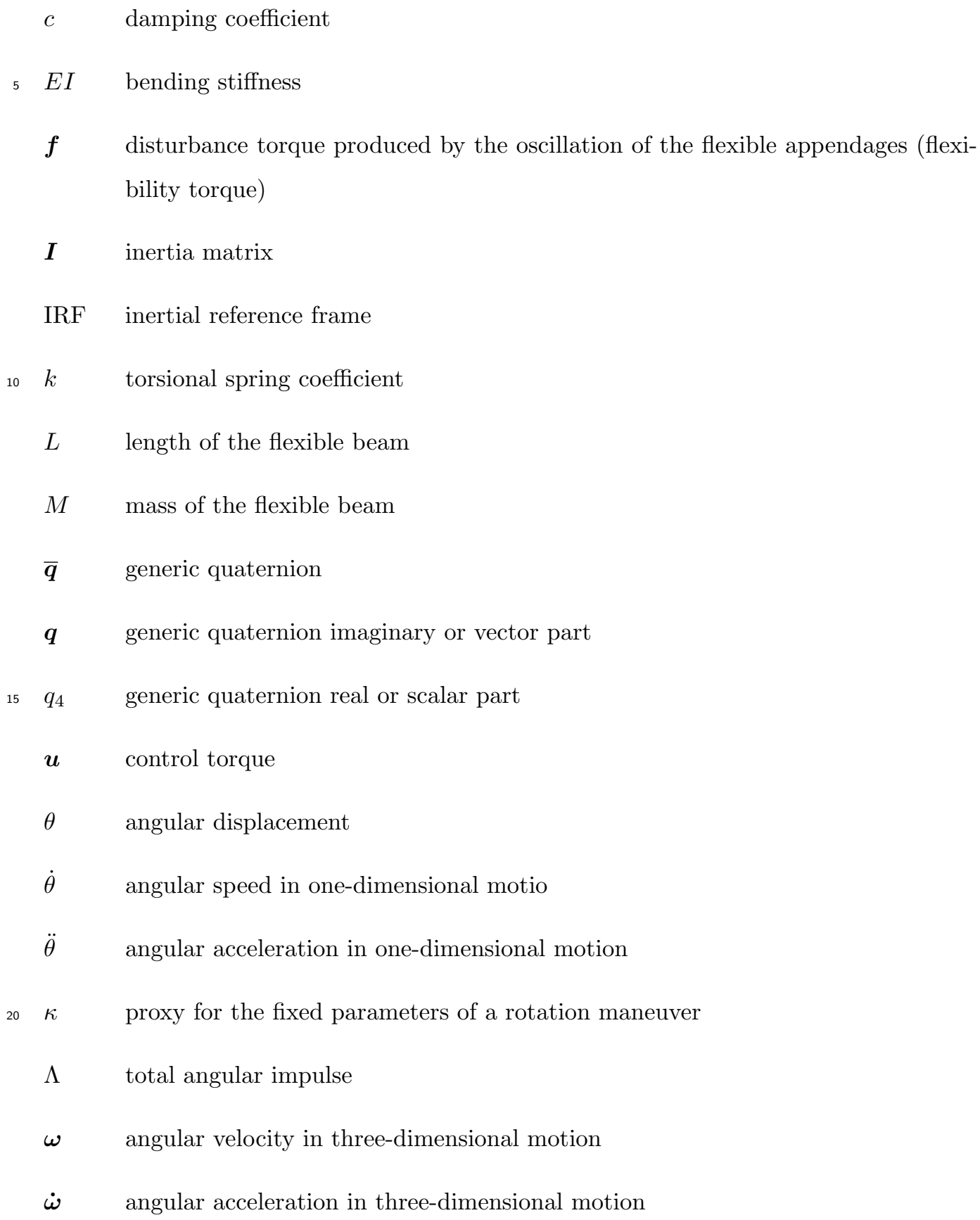




\section{Introduction}

Advances in space technology have led to the development of new spacecraft concepts. Moreover, the introduction of propulsion technology such as solar sail propulsion means that control needs to consider the effects of large flexible appendages. In addition, new mission concepts require the use of multi-body appendages such as robotic arms. However, such structures which store and release elastic energy can have a detrimental effect on pointing accuracy; moreover, strong oscillations may also have an impact on the structure itself, possibly leading to damages to the structure over time. Thus, the attitude control of spacecraft which employ multi-body or flexible appendages must mitigate this by performing smooth motions that avoid exciting the modes.

The main goal of this paper is to develop an efficient guidance technique that enables the attitude control of spacecraft that minimize the oscillation of spacecraft with multibody and flexible appendages.

The problem of controlling the attitude of flexible spacecraft has been extensively studied in literature. Different control laws have been applied in order to mitigate or compensate the effects of the flexible part during the motion. Many approaches rely

40 on robust control methods. For example, Ref. 1] presents an input-shaping technique for use with constant-amplitude actuators on slewing flexible spacecraft; it is robust to modeling errors in the vibration frequency of the appendages. Also, Ref. [2] presents the application of the super-twisting sliding mode control for the three-axis attitude tracking and vibration suppression of a flexible spacecraft. Another approach consists in estimating the disturbance and canceling it using the control; in Ref. 3 a dynamic disturbance compensator is developed to achieve disturbance rejection of the rigid-flexible coupling effects during attitude maneuvers, together with a robust state feedback controller which is derived by analyzing the dynamic characteristics of the flexible part. Ref. 4 presents a control which employs an extended disturbance observer to achieve high-precision attitude stabilization for a spacecraft in the presence of space environmental disturbances, unmodeled dynamics and elastic vibration of flexible appendages. Smart structures have been been proposed to reduce the effect of mode excitation using active vibration suppression, for example in Ref. [5]; however, smart materials are expensive solutions and can be applied to specific structures only. An approach based on optimal control is stud- 
55 ied in Ref. [6], where the attitude stabilization of a flexible spacecraft is achieved using a linear quadratic regulator. Finally, Ref. 7] deals with intelligent control techniques: a neuro-controller is used to determine optimal control laws to minimize the strain energy of flexible arrays during the maneuvers.

While the majority of control methods try to cancel the torque induced by the flexible part to improve pointing and robustness, other approaches use the guidance to plan a smooth motion first, which alleviates the need for a complex control structure. In order to measure the smoothness of a generic motion, it is necessary to introduce a physical quantity which is suitable to represent it. Ref. [8] shows that smoothness can be quantified as a function of jerk, i.e. the time derivative of acceleration, while Ref. [9] 65 compares the minimum jerk, snap and crackle trajectories, finding that the optimization of a smoothness-based cost function for a given trajectory is equivalently achieved by minimizing the jerk. Inspired by these works, the attitude guidance system is designed here so as to reduce the angular jerk as much as possible without exceeding the maximum time allowed for a given maneuver. It is shown in Ref. [10] that the time-optimal threeaxis reorientation maneuver is a bang-bang rotation in all three control components, instead of an eigenaxis rotation about a chosen control axis, which provides the minimum angular path instead. This is due to the fact that the complex geometry of the threeaxis bang-bang has substantial nutational components which provide more torque along the reorientation axis, thus allowing to complete the maneuver in less time. However, a time-optimal bang-bang motion cannot be smoothed without changing the time, so the bang-off-bang structure is considered here. As the bang-off-bang control profile has infinite jerk, it is unsuitable for the control of flexible structures, so a solution proposed by Ref. [1] simply substitutes the bang-off-bang profile with a smoothed curve based on the trigonometric function versine. However, the simple substitution of the steps so of the bang-off-bang maneuver with smooth curves has the drawback of increasing the total time of the maneuver. A new analytical application of such smoothing technique is developed in this paper: when substituting the the steps of the bang-off-bang maneuver with smooth curves, the "off" part of acceleration is reduced to zero and the phases of maximum acceleration are increased, so as to to keep the same displacement and total time of the original bang-off-bang maneuver while smoothing the motion. 
In Refs. [12] and [13] some geometrical smoothing techniques are derived to reduce the excitation of flexible degrees of freedom. First the authors exploit such techniques in order to find a reference angular trajectory; then they use the inverse kinematics to get the three-dimensional (3-D) reference velocity and acceleration, which are then used to determine a pre-planned feed-forward torque to track the spacecraft trajectory. Also, in Ref. [14 a torque profile is fed forward to a controller in order to perform a planned smooth attitude slew maneuver. Ref. 15] presents a method to determine analytical acceleration profiles for single-axis, spin-to-spin slew maneuvers under a finite-jerk constraint. In Ref. [16] an optimization method is used to generate smooth and approximate time-optimal trajectories for attitude maneuvers by minimizing a cost function which takes into account the maneuver duration and the integral of the squared control torque derivatives; however, such methods are numerically solved and computationally expensive relative to the proposed analytical approach of this paper.

The behavior of appendage-like flexible structures is well described in the literature. For example, Ref. [17] provides a model of flexible thin beams in multi-body systems, Ref. [18] presents a general model for flexible multi-body systems based on Kane's method and Ref. [6] shows how to model the dynamics of a flexible spacecraft through Lagrangian mechanics. However, modeling flexible parts as disturbance torques in attitude dynamics is often limited to particular structure cases. For example, in Ref. [19] the flexible appendages are approximated as homogeneous cantilever beams symmetric with respect to the rotation axes, so that only anti-symmetric deformations occur during rotation. In Ref. [7 the spacecraft has a very simple geometry, where the solar arrays are symmetrical with respect to the main body. In Ref. 20] another mathematical model is derived for the attitude dynamics of a spacecraft with flexible appendages, focusing on a spacecraft composed of a rigid body with a long flexible boom with constant cross-sectional properties; the authors developed a complete model for the attitude dynamics which takes into account both the rigid-body dynamics and the oscillatory dynamics of the flexible appendage (in this case a thin beam) by means of the principle of virtual work. Finally, Ref. 21] models the behavior of a satellite with two symmetric panels by means of Kane's dynamics, but considering panels as rigid, with flexible hinges.

A number of possible ways to model a flexible structure exist. Among them, the Ritz 
method, the finite element method and the lumped-parameter method are examples of widely used techniques (Refs. 22] and [23]). For testing the algorithms presented in this paper, any of these models can be employed. The lumped-parameter method is chosen here since not only can it provide an approximate model for flexible appendages, but it also can be used to represent a range of multi-body structures, from boom antennas (modeled as a large number of lumped elements) to rigid multi-body structures such as deployables and robotic arms (which can be represented by fewer lumped element models). This model does not require any specific position or orientation of the appendages with respect to the spacecraft main body or the rotation axis - apart from the alignment with the center of mass of the spacecraft - and it does not require the assumptions of homogeneity and isotropy, provided that the material can be considered perfectly elastic. Once the behavior of the flexible/multi-body structure is modeled, the effects of the oscillation are treated as a disturbance torque to the spacecraft. The resulting model is simple to implement and can easily be extended if multiple flexible parts are present. This model requires the assumption of small elastic deformations, the damping coefficient and the elasticity properties as parameters.

The paper develops as follows. In Sec. 2, a simple model of a thin, flexible structure is derived through the lumped-parameter method and its 3-D behavior is modeled, in order to show how it interacts with the spacecraft main body during the motion. In Sec. 3, the guidance law is developed by means of a trigonometric smoothing technique whose aim is to reduce the sharpness of a generic bang-off-bang rotation. This is first developed for one-dimensional (1-D) rotations, then extended to 3-D maneuvers by means of two approaches: (i) by performing a smooth 1-D rotation about the Euler axis and (ii) by applying the 1-D smoothing technique about each rotation axis separately and combining them through the inverse kinematics equations. In Sec. 4 two simulations are carried out: the first one deals with a planar (1-D) bang-off-bang rotation subjected to the above-mentioned trigonometric smoothing technique, while in the second one the actual behavior of the two approaches for the 3-D smooth guidance is simulated in two different test cases and the results of each of them are compared, in order to show the advantages and disadvantages of the analytical method. 


\section{Modeling flexible structures}

\subsection{Discretization of a thin beam}

Being necessary to have a simple structural flexible model, the lumped-parameter method is used to discretize the 1-D behavior of a thin flexible beam (i.e. bending in one plane only), as it is shown in Fig. 1. which can represent a long flexible appendage such as a boom antenna or a solar panel. This model is also especially well-suited for modeling a multi-body spacecraft structure, such as a robotic arm. The beam has mass $M$, bending stiffness $E I$ and length $L$, and it is attached to the spacecraft main body through a hinge of torsional spring coefficient $k_{r}$ and damping coefficient $c_{r}$. All the structural parameters are known and the mass and the bending stiffness of the beam are assumed to be uniform, i.e. the structure is homogeneous.

The flexible beam has been discretized into two rigid segments joined by a fictitious hinge located at the middle of the beam. The segments are massless, thus two masses have been placed in the middle of each segment to represent the mass of the whole beam.

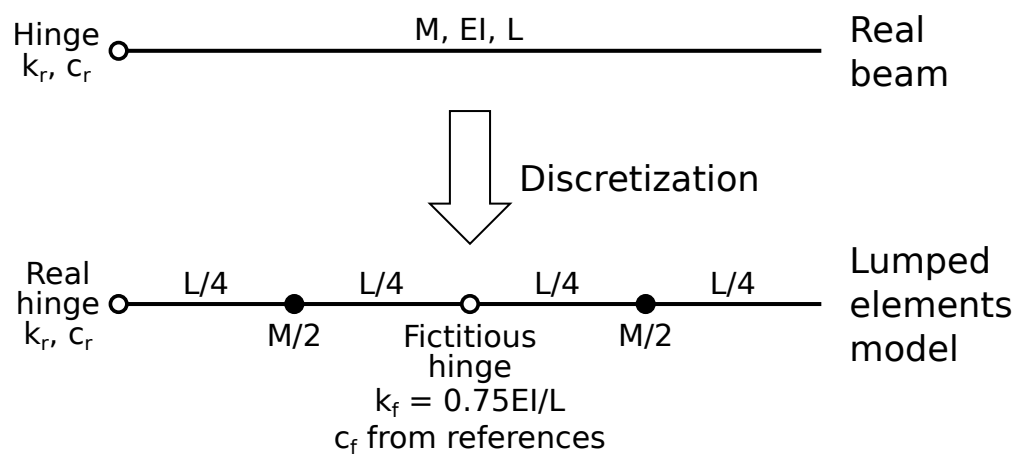

Figure 1: Discretization of a thin beam.

The fictitious hinge has its own torsional spring coefficient $k_{f}$ and damping coefficient $c_{f}$, representing the flexibility and the damping capability of the full beam. $k_{f}$ can be recovered from the bending stiffness of the beam under two assumptions:

- the contribution of the shear stiffness is small enough to be neglected without introducing a significant error;

- the inertia forces acting on each infinitesimal point of the structure are considered uniform along the beam and may be represented by a uniform distributed load; 
this is more true as the length of the beam is shorter, meaning that the accuracy of the model increases by increasing the number of fictitious hinges and masses.

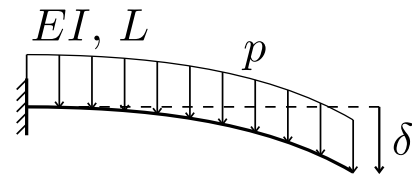

(a) Inertia load.

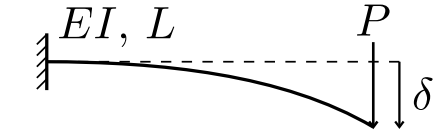

(b) Equivalent concentrated load.

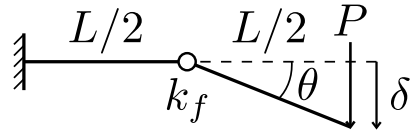

(c) Equivalent hinge.

Figure 2: Model of the bending stiffness of the fictitious hinge. sented as a beam subjected to a uniformly-distributed force $p$, as it is shown in Fig. 2a Such force produces a displacement $\delta$ at the end of the beam that can be computed through Castigliano's method (Ref. [24, p. 330]), obtaining:

$$
\delta=\frac{p L^{4}}{8 E I}
$$

The distributed load $p$ is then converted into a concentrated one $P$ which is located at the tip of the beam and produces the same displacement of the distributed load:

$$
\delta=\frac{P L^{3}}{3 E I}
$$

as it is shown in Fig. 2b. By equating the displacements it is possible to get a relation between $p$ and $P$ :

$$
P=\frac{3}{8} p L
$$

Now the beam is discretized into two rigid segments coupled through a joint, as it is shown in Fig. 2c Being $\tau$ the torque caused by the concentrated load $P$ at the tip and 180 $\theta$ the bending angle of the second rigid segment with respect to its equilibrium position, the torsional spring coefficient of the hinge $k_{f}$ is then given by:

$$
k_{f}=\frac{\tau}{\theta}
$$


with

$$
\tau=\frac{1}{2} P L
$$

and, with the reasonable assumption of small angle bending,

$$
\theta \approx \sin \theta=\frac{\delta}{L / 2}
$$

the torsional spring coefficient is obtained:

$$
k_{f}=\frac{3 E I}{4 L}
$$

185

This coefficient replaces the internal flexibility of the beam with a single fictitious hinge, allowing to treat the structure as two joint rigid segments. Unfortunately, no analytic expression was found to determine the damping coefficient $c_{f}$ of the fictitious hinge, thus its value must be found empirically through on-ground testing or other references, for example similar structures or more accurate structural analyses such as the finite element method.

\subsection{1-D dynamics of the structure}

The dynamic behavior of the flexible system is studied exploiting Lagrangian mechanics. An array of $N$ beams is now considered, which are discretized into lumped elements according to the method presented in the previous section. As each beam is discretized into two elements, a total of $2 N$ lumped elements will be present, each one composed by a hinge (real or fictitious), a rigid beam segment and a point mass.

First, three functions are computed:

$$
\begin{aligned}
T & =\sum_{i=1}^{2 N} \frac{1}{2} I_{i}\left(\dot{\theta}_{i}+\dot{\theta}_{B}\right)^{2} & & \text { kinetic energy } \\
V & =\sum_{i=1}^{2 N} \frac{1}{2} k_{i}\left(\theta_{i}-\theta_{i-1}\right)^{2} & & \text { potential energy } \\
D & =\sum_{i=1}^{2 N} \frac{1}{2} c_{i}\left(\dot{\theta}_{i}-\dot{\theta}_{i-1}\right)^{2} & & \text { dissipation function }
\end{aligned}
$$

where, referring to Fig. 3 
- $I_{i}=m_{i} r_{i}^{2}$ is the moment of inertia of the $i$-th lumped mass $m_{i}$, located at a distance $r_{i}$ from the rotation axis;

- $k_{i}$ and $c_{i}$ are the spring coefficient and the damping coefficient of the $i$-th hinge respectively;

- $\theta_{i}$ and $\dot{\theta}_{i}$ are the $i$-th angular displacement and angular speed respectively with respect to the equilibrium position;

- $\dot{\theta}_{B}$ is the angular speed of the body reference frame (BRF) with respect to the inertial reference frame (IRF), i.e. the angular speed of the spacecraft rigid body;

- $\theta_{0}$ and $\dot{\theta}_{0}$ are the angular position and speed of the equilibrium position with respect to BRF, which are assumed to be zero (i.e. the equilibrium position is aligned with $\left.\hat{\boldsymbol{x}}_{B}\right)$.

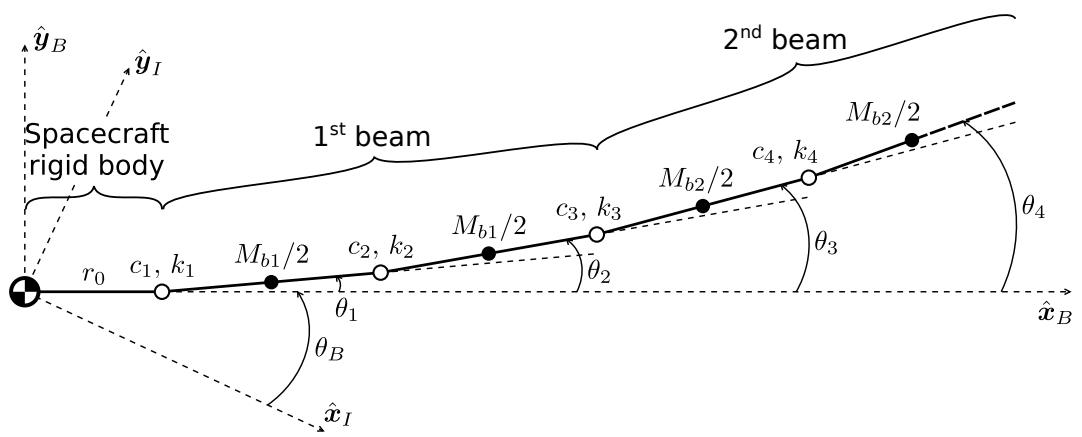

Figure 3: Example of a lumped-parameter model of an array of two beams undergoing a rotational motion.

Now it is possible to compute the Lagrangian equations for the lumped elements, as in Ref. [6, p. 35]:

$$
\frac{d}{d t} \frac{\partial T}{\partial \dot{\theta}_{i}}-\frac{\partial T}{\partial \theta_{i}}+\frac{\partial V}{\partial \theta_{i}}+\frac{\partial D}{\partial \dot{\theta}_{i}}=0 \quad i=1, \ldots, 2 N
$$

Another Lagrangian equation must be added in order to take into account the external contributions to the motion of the flexible structure:

$$
\frac{d}{d t} \frac{\partial T}{\partial \dot{\theta}_{B}}-\frac{\partial T}{\partial \theta_{B}}+\frac{\partial V}{\partial \theta_{B}}+\frac{\partial D}{\partial \dot{\theta}_{B}}=f
$$


where $f$ is the flexibility torque: a boundary reaction torque which is produced by the 215 motion of the flexible structure on the spacecraft main body.

By solving Eq. 12, it is possible to find the contribution of the flexibility to the total torque applied on the spacecraft:

$$
f=\sum_{i=1}^{N} m_{i} r_{i}^{2}\left(\ddot{\theta}_{i}+\ddot{\theta}_{B}\right)
$$

Assembling all the Lagrangian equations allows to write the equations of motion of the system in a matrix form:

$$
\boldsymbol{I} \ddot{\boldsymbol{\theta}}+\boldsymbol{K} \boldsymbol{\theta}+\boldsymbol{C} \dot{\boldsymbol{\theta}}=\boldsymbol{\tau}
$$

220 with

$$
\begin{aligned}
\boldsymbol{I} & =\left[\begin{array}{cccccc}
\sum_{i=1}^{2 N} m_{i} r_{i}^{2} & m_{1} r_{1}^{2} & m_{2} r_{2}^{2} & m_{3} r_{3}^{2} & \ldots & m_{2 N} r_{2 N}^{2} \\
m_{1} r_{1}^{2} & m_{1} r_{1}^{2} & 0 & 0 & \ldots & 0 \\
m_{2} r_{2}^{2} & 0 & m_{2} r_{2}^{2} & 0 & \ldots & 0 \\
m_{3} r_{3}^{2} & 0 & 0 & m_{3} r_{3}^{2} & \ldots & 0 \\
\vdots & \vdots & \vdots & \vdots & \ddots & \vdots \\
m_{2 N} r_{2 N}^{2} & 0 & 0 & 0 & \ldots & m_{2 N} r_{2 N}^{2}
\end{array}\right] \\
\boldsymbol{K} & =\left[\begin{array}{ccccccc}
0 & 0 & 0 & 0 & \ldots & 0 \\
0 & k_{1}+k_{2} & -k_{2} & 0 & \ldots & 0 \\
0 & -k_{2} & k_{2}+k_{3} & -k_{3} & \ldots & 0 \\
0 & 0 & -k_{3} & k_{3}+k_{4} & \ldots & 0 \\
\vdots & \vdots & \vdots & \vdots & \ddots & -k_{2 N} \\
0 & 0 & 0 & 0 & -k_{N} & k_{2 N}
\end{array}\right] \\
\boldsymbol{C} & =\left[\begin{array}{ccccccc}
0 & 0 & 0 & 0 & \ldots & 0 \\
0 & c_{1}+c_{2} & -c_{2} & 0 & \ldots & 0 \\
0 & -c_{2} & c_{2}+c_{3} & -c_{3} & \ldots & 0 \\
0 & 0 & -c_{3} & c_{3}+c_{4} & \ldots & 0 \\
\vdots & \vdots & \vdots & \vdots & \ddots & -c_{2 N} \\
0 & 0 & 0 & 0 & -c_{N} & c_{2 N}
\end{array}\right]
\end{aligned}
$$


being $\boldsymbol{I}$ the inertia matrix, $\ddot{\boldsymbol{\theta}}=\left[\begin{array}{lllll}\ddot{\theta}_{B} & \ddot{\theta}_{1} & \ddot{\theta}_{2} & \ldots & \ddot{\theta}_{2 N}\end{array}\right]^{T}$ a vector collecting the angular accelerations of the main body and the lumped elements, $\boldsymbol{K}$ the stiffness matrix, $\boldsymbol{C}$ the ${ }_{225}$ damping matrix and $\boldsymbol{\tau}=\left[\begin{array}{cccc}f & 0 & 0 & \ldots\end{array}\right]^{T}$ the vector of external loads.

Eq. (14) represents thus a two-way coupling between the motion of the rigid body of the spacecraft (represented by the term $\ddot{\theta}_{B}$ ) and the motion of the flexible structure, whose effect on the main body is represented by $f$.

\subsection{3-D dynamics of the structure}

In order to extend this approach to the 3-D case, it is first necessary to define a new reference frame which is more suitable for describing the behavior of the flexible part. This new reference frame is called appendage reference frame $(\mathrm{ARF})$ and is defined such that $\hat{\boldsymbol{x}}_{A}$ is aligned with the main axis of the appendage when it is in equilibrium, while $\hat{\boldsymbol{y}}_{A}$ and $\hat{\boldsymbol{z}}_{A}$ are left to the free choice of the designers, provided that they follow the convention of the right hand rule. It is also required that the origin of the ARF frame coincides with the center of mass of the spacecraft, which means that the principal axis of the flexible structure must be aligned with the center of mass of the spacecraft. A rotation matrix is given to convert this reference frame to BRF and vice-versa: $\boldsymbol{R}_{B \leftarrow A}=\boldsymbol{R}_{A \leftarrow B}^{T}$.

In order to manage a 3 -D deformation of the flexible appendage, its dynamic behavior is decomposed into two bending deformations, each one along the $\hat{\boldsymbol{y}}_{A}$ and $\hat{\boldsymbol{z}}_{A}$ axes of ARF, and a torsional contribution about $\hat{\boldsymbol{x}}_{A}$ due to the torsional inertia of the appendage. This approach offers a good solution because of the slimness of the beam, since gyroscopic effects do not significantly affect slim beams.

First, the same discretization process seen in Sec. 2.1 is applied about each axis $\hat{\boldsymbol{y}}_{A}$ and $\hat{\boldsymbol{z}}_{A}$ separately so as to obtain the same series of masses and hinges on the beam. The $i$-th hinge along $\hat{\boldsymbol{y}}_{A}$ and the one along $\hat{\boldsymbol{z}}_{A}$ lie to the same position along the appendage, but they may have different mechanical properties if the structure is anisotropic.

The position $\boldsymbol{r}_{i}$ of each lumped mass $m_{i}$ in the BRF is given by:

$$
\boldsymbol{r}_{i}=\boldsymbol{R}_{B \leftarrow A} r_{i}\left[\begin{array}{lll}
1 & 0 & 0
\end{array}\right]^{T}
$$

If the alignment of the rotation axis $\hat{\boldsymbol{\omega}}$ with respect to the body frame is known 250 (rate-integrating gyros can provide this piece of information), it is possible to determine 
the distance of the $i$-th mass from that axis so as to compute its moment of inertia. As shown in Fig. 4 , that distance is

$$
d_{i}=r_{i} \sin \alpha
$$

being $\alpha$ the angle between the main axis of the appendage and the axis of rotation:

$$
\alpha=\arccos \left(\frac{\boldsymbol{\omega} \cdot \boldsymbol{r}_{i}}{\omega r_{i}}\right)=\arccos \left(\hat{\boldsymbol{\omega}} \cdot \boldsymbol{R}_{B \leftarrow A}\left[\begin{array}{lll}
1 & 0 & 0
\end{array}\right]^{T}\right)
$$

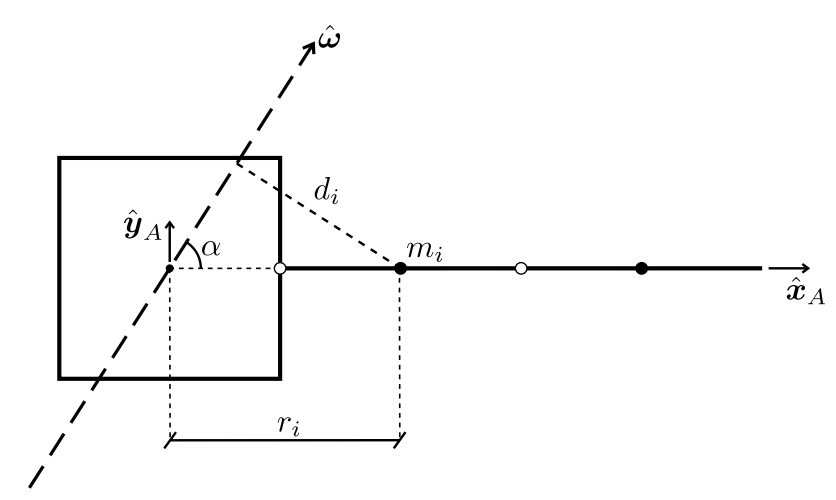

Figure 4: Appendage with three-dimensional rotation.

In order to extend Eq. 13 to the 3-D case, the angular accelerations of the lumped masses about $\hat{\boldsymbol{y}}_{A}$ and $\hat{\boldsymbol{z}}_{A}$ and the angular acceleration of the spacecraft are used. Therefore, instead of the planar angular accelerations, now the 3-D accelerations of the lumped masses and the spacecraft are used:

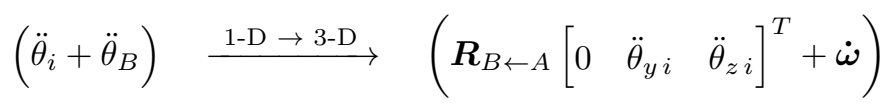

Moreover, a contribution due to the torsional inertia is added. Once the spacecraft angular acceleration $\dot{\boldsymbol{\omega}}$ is converted into $\mathrm{ARF}$, it can be recovered by multiplying the polar mass moment of inertia $I_{p i}$ by the component of acceleration that is aligned with $\hat{\boldsymbol{x}}_{A}$, i.e. the first element of the vector; the result is then reconverted into BRF. The 
following equation is thus obtained:

$$
\boldsymbol{f}=\sum_{i=1}^{N}\left[m_{i} d_{i}^{2}\left(\boldsymbol{R}_{B \leftarrow A}\left\{\begin{array}{c}
0 \\
\ddot{\theta}_{y i} \\
\ddot{\theta}_{z i}
\end{array}\right\}+\left\{\begin{array}{c}
\dot{\omega}_{x} \\
\dot{\omega}_{y} \\
\dot{\omega}_{z}
\end{array}\right\}\right)+\boldsymbol{R}_{B \leftarrow A} I_{p i}\left[\begin{array}{ccc}
1 & 0 & 0 \\
0 & 0 & 0 \\
0 & 0 & 0
\end{array}\right] \boldsymbol{R}_{A \leftarrow B}\left\{\begin{array}{c}
\dot{\omega}_{x} \\
\dot{\omega}_{y} \\
\dot{\omega}_{z}
\end{array}\right\}\right]
$$

Note that while the torsional inertia is kept into account, the torsional deformation of the structure is not.

\section{Low-jerk attitude guidance and control}

In this section, a controlled rest-to-rest maneuver is considered, which is given in terms of desired displacement (angular distance between the desired position and the current one) and required time for the maneuver. In order to perform such maneuver, it is necessary to design a suitable trajectory which satisfies the requirements while being constrained by the physical properties of the spacecraft and by keeping the excitation of the flexible structures to a minimum. Such trajectory will be expressed as an acceleration profile that shall be used to feed a suitable control.

\subsection{1-D maneuvers}

The versine control profile is a simple smooth approximation of the bang-off-bang control profile (Fig. 5) with a finite jerk value by means of the versine trigonometric function. The versine is defined as the difference between 1 and the cosine of a given angle:

$$
\operatorname{versin}(\theta):=1-\cos (\theta)
$$

This function is used to smoothen each step of the bang-bang control. As its derivative is zero at the origin, it is well suitable to have smooth start and smooth end.

Once the quick changes of acceleration of the bang-off-bang profile are substituted by versine-shaped curves, as it is possible to see in Fig. 6 it is necessary to define some quantities: 


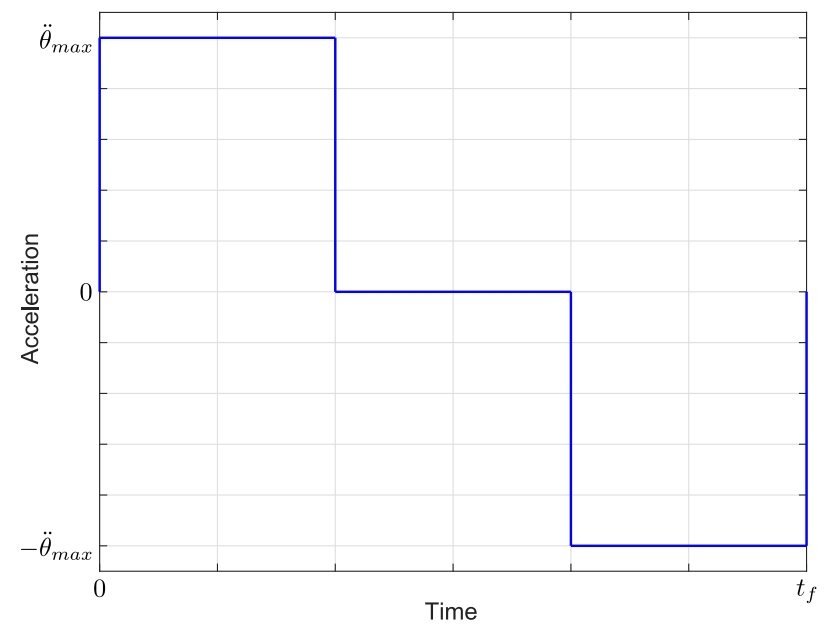

Figure 5: Profile of the bang-off-bang maneuver.

- $t_{v}$ is the time of each application of the versine function;

- $t_{m}$ is the time of constant acceleration between the increasing and the decreasing parts of the versine function;

- $t_{n a}$ is the time of constant velocity between the positive and negative acceleration phases;

- $t_{f}=4 t_{v}+2 t_{m}+t_{n a}$ is the total time of the acceleration profile, which is less than or equal to the duration of the maneuver.

It is clear that, in order to increase the smoothness of the maneuver, it is necessary to increase $t_{v}$. The best way to achieve this goal without increasing the total time of flight is to reduce $t_{n a}$ to zero so that the end of the positive acceleration phase coincides to the start of the negative one, as it is possible to see in Fig. 7. Then the total time of the acceleration profile becomes:

$$
t_{f}=4 t_{v}+2 t_{m}
$$

At this point, it is possible to see that a stationary point is present where the acceleration switches sign. Such ripple is a consequence of two consecutive and opposite versine curves and has no usefulness, therefore it may be better to replace the part of the curve 


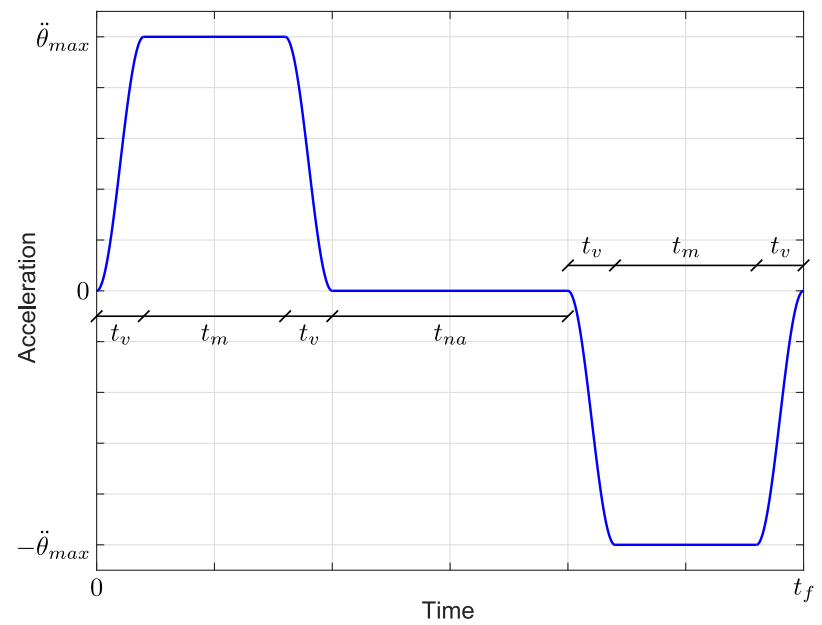

Figure 6: Profile of the versine-smoothed maneuver.

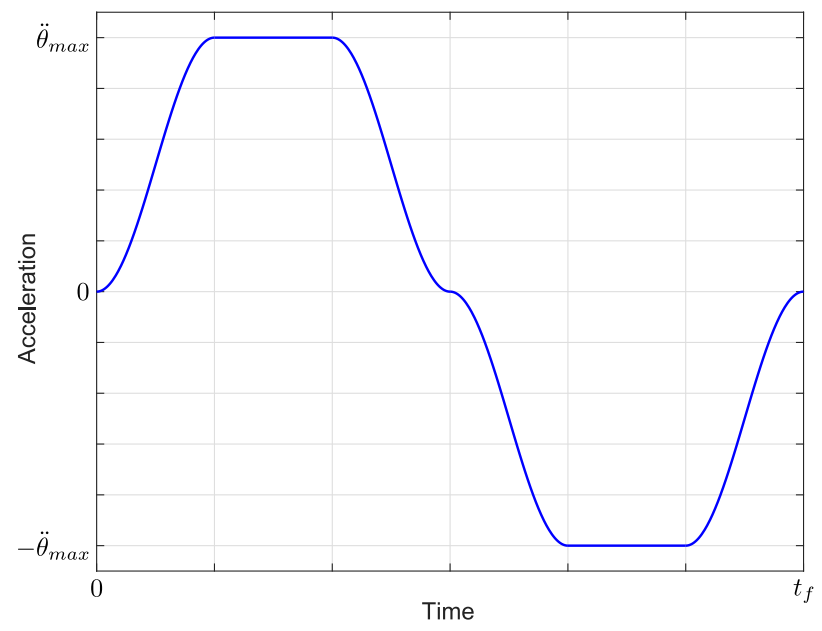

Figure 7: Profile of the versine-smoothed maneuver without constant-velocity part.

between the highest and the lowest peaks of acceleration with a cosine, which does not present any distortion near zero. The final profile is shown in Fig. 8 .

Given a maximum available acceleration $\ddot{\theta}_{\max }$, the actual control acceleration is therefore given by 


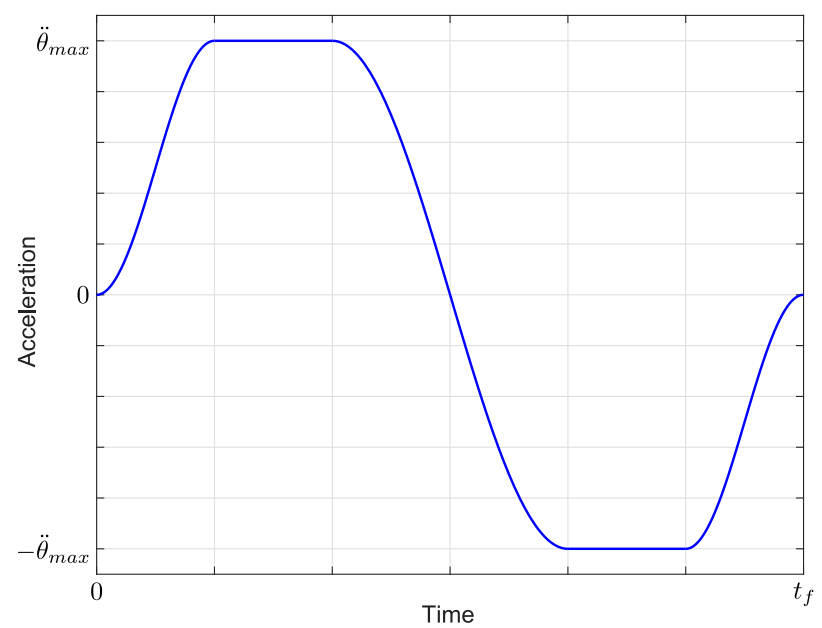

Figure 8: Profile of the versine/cosine-smoothed maneuver.

$$
\begin{array}{ll}
\ddot{\theta}(t)=\frac{1}{2} \ddot{\theta}_{\text {max }} \operatorname{versin}\left(\pi \frac{t}{t_{v}}\right) & 0 \leq t<t_{v} \\
\ddot{\theta}(t)=\ddot{\theta}_{\text {max }} & t_{v} \leq t<t_{m}+t_{v} \\
\ddot{\theta}(t)=\ddot{\theta}_{\text {max }} \cos \left(\frac{\pi}{2} \frac{t-t_{m}-t_{v}}{t_{v}}\right) & t_{m}+t_{v} \leq t<t_{m}+3 t_{v} \\
\ddot{\theta}(t)=-\ddot{\theta}_{\text {max }} & t_{m}+3 t_{v} \leq t<2 t_{m}+3 t_{v} \\
\ddot{\theta}(t)=-\frac{1}{2} \ddot{\theta}_{\text {max }} \operatorname{versin}\left(\pi \frac{t-2 t_{m}-4 t_{v}}{t_{v}}\right) & 2 t_{m}+3 t_{v} \leq t<t_{f}
\end{array}
$$

It is now necessary to correlate the parameters of the maneuver shaping, $t_{v}$ and $t_{m}$, to the constraints of the maneuver and the spacecraft: the final time $t_{f}$, the desired displacement $\theta_{d}$, the maximum available control torque $u_{\max }$ and the inertia $I$. Under the assumption of zero initial position, $\theta(0)=0$, the displacement can be obtained from the double integration of the acceleration profile:

$$
\begin{aligned}
\theta=2 \ddot{\theta}_{\max }\left[\iint_{0}^{t_{v}} \frac{1}{2} \operatorname{versin}\left(\pi \frac{t}{t_{v}}\right) d t d t\right. & +\iint_{t_{v}}^{t_{m}+t_{v}} d t d t+ \\
& \left.+\iint_{t_{m}+t_{v}}^{t_{m}+2 t_{v}} \cos \left(-\pi \frac{t-t_{m}-t_{v}}{2 t_{v}}\right) d t d t\right]
\end{aligned}
$$


Defining

$$
\kappa:=\frac{\theta_{d}}{\ddot{\theta}_{\max }}=\frac{\theta_{d} I}{u_{\max }}
$$

as a proxy for the fixed parameters and substituting $t_{m}=\frac{1}{2} t_{f}-2 t_{v}$, the following equation is obtained:

$$
\left(\frac{6}{\pi^{2}}-\frac{1}{2}\right) t_{v}^{2}-\frac{1}{2} t_{f} t_{v}+\frac{1}{4} t_{f}^{2}=\kappa
$$

310

which must be solved with the constraints of $t_{f}, \kappa>0$ and $0 \leq t_{v} \leq \frac{1}{4} t_{f}$.

For given values of $\kappa$ and $t_{f}$, that equation is satisfied when:

$$
t_{v}=\frac{\pi \sqrt{\left(3 \pi^{2}-24\right) t_{f}^{2}+\left(96-8 \pi^{2}\right) \kappa}-\pi^{2} t_{f}}{2\left(\pi^{2}-12\right)}
$$

It is worth to note that for a given value of $\kappa$ the total time $t_{f}$ is constrained by a lower and an upper bound. The lower bound is given by

$$
t_{f \min }=2 \sqrt{\kappa}
$$

which corresponds to a bang-bang maneuver, where $t_{v}=0$ and $t_{m}=\frac{1}{2} t_{f}$. The bang-bang profile is the time-optimal maneuver, so no maneuver is possible with a lower duration.

The upper bound is given by

$$
t_{f \max }=\frac{4 \pi \sqrt{2}}{\sqrt{3 \pi^{2}+12}} \sqrt{\kappa}
$$

which coincides with $t_{v}=\frac{1}{4} t_{f}$ and $t_{m}=0$. A larger value of $t_{f}$ requires to reduce the maximum acceleration to perform the maneuver correctly: if $t_{f}$ exceeds such limit, a new value for the maximum acceleration must be set as

$$
\ddot{\theta}_{\text {max new }}=\frac{32 \pi^{2} \theta_{d}}{t_{f}^{2}\left(3 \pi^{2}+12\right)}
$$

with $\kappa$ varying accordingly.

This set of equations allows to create a suitable low-jerk acceleration profile for a specific maneuver, providing the desired displacement $\theta_{d}$, in the total time $t_{f}$ and within the maximum acceleration $\ddot{\theta}_{\max }=u_{\max } / I$. First $t_{f}$ must be compared with the bounds 
provided by Eq. (30) and 231); if it is too small, the maneuver is physically infeasible for 325 the spacecraft and it is necessary to increase the time of flight or the maximum available torque; if on the contrary $t_{f}$ is too large, the maximum acceleration $\ddot{\theta}_{\text {max }}$ shall be reduced to a new value given by Eq. (32). After that, the times of the different phases of the maneuver, $t_{v}$ and $t_{m}$, can be found through Eq. 29) and (24) respectively, which in the end allow to design the acceleration profile according to Eq. 25).

It is worth noting that the maximum angular velocity of the smooth motion increases with respect to a bang-off-bang maneuver. This is because The "off" part in the profile is produced not only by the constraint on maximum acceleration, but also by the constraint on maximum angular velocity that the spacecraft can withstand. For this reason, it may be necessary to increase the total time in order to decrease the maximum angular velocity.

335 As it is shown in Ref. [25], this can be done by rescaling the time as $t=\alpha \tau$, where $\tau$ is the new total time and $\alpha$ is a rescaling factor; so if the planned maneuver exceeds the maximum angular velocity, it is possible to rescale the total time so that it respects such constraint.

By integrating Eq. (25) twice it is possible to get analytically the velocity and position 
Phase 1: positive versine-shaped acceleration $\left(0 \leq t<t_{v}\right)$

$$
\begin{aligned}
& \dot{\theta}(t)=\ddot{\theta}_{\max }\left[\frac{t}{2}-\frac{t_{v}}{2 \pi} \sin \left(\pi \frac{t}{t_{v}}\right)\right] \\
& \theta(t)=\ddot{\theta}_{\max }\left\{\frac{t^{2}}{4}-\frac{t_{v}^{2}}{2 \pi^{2}}\left[\cos \left(\pi \frac{t}{t_{v}}\right)-1\right]\right\}
\end{aligned}
$$

Phase 2: positive constant acceleration $\left(t_{v} \leq t<t_{m}+t_{v}\right)$

$$
\begin{aligned}
\dot{\theta}(t) & =\ddot{\theta}_{\max }\left(t-\frac{1}{2} t_{v}\right) \\
\theta(t) & =\ddot{\theta}_{\max }\left[\frac{1}{2} t^{2}-\frac{1}{2} t_{v} t-\left(\frac{1}{\pi^{2}}-\frac{1}{4}\right) t_{v}^{2}\right]
\end{aligned}
$$

Phase 3: cosine-shaped acceleration $\left(t_{m}+t_{v} \leq t<t_{m}+3 t_{v}\right)$

$$
\begin{aligned}
& \dot{\theta}(t)=\ddot{\theta}_{\max }\left\{\left[-\frac{2}{\pi} \cos \left(\frac{\pi}{2} \frac{t-t_{m}}{t_{v}}\right)+\frac{1}{2}\right] t_{v}+t_{m}\right\} \\
& \theta(t)=\ddot{\theta}_{\max }\left\{\left[-\frac{4}{\pi^{2}} \sin \left(\frac{\pi}{2} \frac{t-t_{m}}{t_{v}}\right)+\frac{3}{\pi^{2}}-\frac{1}{4}\right] t_{v}^{2}+t_{m} t+\frac{1}{2} t_{v} t-\frac{1}{2} t_{m}^{2}-\right. \\
&\left.-t_{m} t_{v}\right\}
\end{aligned}
$$

Phase 4: negative constant acceleration $\left(t_{m}+3 t_{v} \leq t<2 t_{m}+3 t_{v}\right)$

$$
\begin{aligned}
\dot{\theta}(t) & =\ddot{\theta}_{\max }\left(-t+2 t_{m}+\frac{7}{2} t_{v}\right) \\
\theta(t) & =\ddot{\theta}_{\max }\left[-\frac{1}{2} t^{2}+2 t_{m} t+\frac{7}{2} t_{v} t+\left(\frac{7}{\pi^{2}}-\frac{19}{4}\right) t_{v}^{2}-t_{m}^{2}-4 t_{m} t_{v}\right]
\end{aligned}
$$

Phase 5: negative versine-shaped acceleration $\left(2 t_{m}+3 t_{v} \leq t<t_{f}\right)$

$$
\begin{aligned}
\dot{\theta}(t) & =\ddot{\theta}_{\max }\left\{-\frac{1}{2} t+\left[\frac{1}{2 \pi} \sin \left(\pi \frac{t-2 t_{m}-4 t_{v}}{t_{v}}\right)+2\right] t_{v}+t_{m}\right\} \\
\theta(t) & =\ddot{\theta}_{\max }\left\{-\frac{1}{4} t^{2}+t_{m} t+2 t_{v} t-\left[\frac{1}{2 \pi^{2}} \cos \left(\pi \frac{t-2 t_{m}-4 t_{v}}{t_{v}}\right)-\frac{13}{2 \pi^{2}}+\right.\right. \\
+ & \left.\left.\frac{5}{2}\right] t_{v}^{2}-t_{m} t_{v}\right\}
\end{aligned}
$$

\subsection{3-D maneuvers}

In order to work on the 3 -D case, it is necessary to use a 3-D representation of the maneuver. Quaternions are well suitable to represent 3-D attitude and spatial rotations, so a quaternion representation is adopted. A generic quaternion $\overline{\boldsymbol{q}}$ is composed by an 
imaginary or vector part $\boldsymbol{q}$ and a real or scalar part $q_{4}$ as follows:

$$
\overline{\boldsymbol{q}}=\left[\begin{array}{c}
\boldsymbol{q} \\
q_{4}
\end{array}\right], \quad \boldsymbol{q}=\left[\begin{array}{l}
q_{1} \\
q_{2} \\
q_{3}
\end{array}\right]
$$

The quaternion error of the overall maneuver can be found as

$$
\overline{\boldsymbol{q}}_{e}=\overline{\boldsymbol{q}}_{d}^{-1} \otimes \overline{\boldsymbol{q}}_{0}
$$

where $\overline{\boldsymbol{q}}_{d}$ is the desired final quaternion, $\overline{\boldsymbol{q}}_{0}$ is the quaternion representing the initial position, $\otimes$ represents the quaternion product, and the inverse of a quaternion is obtained as

$$
\overline{\boldsymbol{q}}^{-1}=\frac{1}{\|\overline{\boldsymbol{q}}\|^{2}}\left[\begin{array}{c}
-\boldsymbol{q} \\
q_{4}
\end{array}\right]
$$

350 being $\|\overline{\boldsymbol{q}}\|=\sqrt{q_{1}^{2}+q_{2}^{2}+q_{3}^{2}+q_{4}^{2}}$ the norm of the quaternion.

It is worth to note that it is always possible to change the reference frame of the maneuver such that in the new frame the initial angular position is zero.

\subsubsection{Smoothing of the eigenaxis rotation}

Euler's rotation theorem states that any 3-D rotation of a rigid body about a fixed point from any initial orientation to any final orientation is equivalent to a single rotation about an axis that runs through the fixed point (Ref. [26, p. 329]). Such axis is stationary in an inertial reference frame and is called Euler axis or eigenaxis.

The eigenaxis rotation, being about an inertial fixed axis, can be considered a 1-D rotation in a reference frame which has the Euler axis as one of its main axes. Owing to this, the 1-D smoothing technique developed in the previous section can be applied directly.

It is worth to note, however, that as the Euler axis is not a principal axis of inertia, a torque about this axis does not only result in an acceleration about the same, and therefore additional control torques around the other axes are needed. 

resentation in four numbers, where the vector part represents the eigenaxis $\hat{\boldsymbol{e}}$ and the scalar part the angle $\theta$ :

$$
\overline{\boldsymbol{q}}=\left[\begin{array}{c}
\sin \frac{\theta}{2} \hat{\boldsymbol{e}} \\
\cos \frac{\theta}{2}
\end{array}\right]
$$

In other words, quaternions define the rigid-body attitude as an Euler-axis rotation. This means that the vector of the imaginary part is aligned with the Euler axis while the real part is associated to the Euler angle, so it is possible to compute the Euler axis $\hat{e}$ and the desired angle $\theta_{d}$ of a given maneuver from the quaternion error as follows:

$$
\begin{aligned}
& \hat{\boldsymbol{e}}=\csc \frac{\theta_{d}}{2} \boldsymbol{q}_{e}=\frac{\boldsymbol{q}_{e}}{\left\|\boldsymbol{q}_{e}\right\|} \\
& \theta_{d}=2 \arccos q_{e 4}
\end{aligned}
$$

Once the angular displacement $\theta_{d}$ is obtained, it can be smoothed by using the set of Eq. (33): by computing the analytical angular displacement path $\theta(t)$ from $t=0$ to $t=t_{f}$, a 1-D reference displacement $\theta_{r e f}(t)$ is obtained which the spacecraft must 375 follow in order to perform the smooth maneuver. Such 1-D profile of displacement is then converted into a quaternion $\overline{\boldsymbol{q}}_{r e f}(t)$ which represents a 3-D reference maneuver, as follows:

$$
\begin{aligned}
& \boldsymbol{q}_{r e f}(t)=\sin \frac{\theta_{r e f}(t)}{2} \hat{\boldsymbol{e}} \\
& q_{r e f 4}(t)=\cos \frac{\theta_{\text {ref }}(t)}{2}
\end{aligned}
$$

A reference speed $\dot{\theta}_{\text {ref }}(t)$ about the Euler axis can also be obtained by computing the angular speed $\dot{\theta}(t)$ through Eq. 33. It is then converted into a 3-D profile of velocity as

$$
\boldsymbol{\omega}_{\text {ref }}(t)=\dot{\theta}_{\text {ref }}(t) \hat{\boldsymbol{e}}
$$

The 3-D profiles of position $\overline{\boldsymbol{q}}_{r e f}(t)$ and velocity $\boldsymbol{\omega}_{r e f}(t)$ can then be exploited for the smooth maneuver. 


\subsubsection{Smoothing of a generic 3-D maneuver}

Even if the eigenaxis rotation can be used to perform maneuvers in the 3-D rotation group, it may be useful to find a way to smooth generic 3-D maneuvers without relying on the specific rotation about the Euler axis.

An approach to create 3-D maneuvers starting from a 1-D smoothing technique is proposed by Ref. [12]. Such method is adopted here to design a fully-3-D maneuver profile that takes into account the nonlinear terms of Euler equations starting from the 1-D analytical smoothing technique developed in the previous section.

First, a smooth profile maneuver is developed for each axis separately, starting from the desired displacement $\theta_{d}$ about each axis and the same total maneuver time $t_{f}$ and using Eq. (33). Three profiles of position are then obtained:

$$
\boldsymbol{\theta}_{\text {ref }}(t)=\left[\begin{array}{lll}
\theta_{\text {ref } x}(t) & \theta_{\text {ref } y}(t) & \theta_{\text {ref } z}(t)
\end{array}\right]^{T}
$$

which can be converted into a quaternion representation in order to obtain the profile of position in terms of quaternions, $\overline{\boldsymbol{q}}_{\text {ref }}(t)$.

Since it is not possible to integrate directly the Euler angles to get the profiles of angular velocities, the kinematic equations of Euler angles are used to find the angular velocity vector $\boldsymbol{\omega}_{r e f}(t)$ starting from the kinematic equations for the set of Euler angles $3-2-1$ :

$$
\dot{\boldsymbol{\theta}}=\frac{1}{\cos \theta_{y}}\left[\begin{array}{ccc}
0 & \sin \theta_{z} & \cos \theta_{z} \\
0 & \cos \theta_{y} \cos \theta_{z} & -\cos \theta_{y} \sin \theta_{z} \\
\cos \theta_{y} & \sin \theta_{y} \sin \theta_{z} & \sin \theta_{y} \cos \theta_{z}
\end{array}\right] \boldsymbol{\omega}
$$

Such equations are inverted to find the reference angular velocity from the derivatives of the reference Euler angles:

$$
\boldsymbol{\omega}_{\text {ref }}(t)=\left[\begin{array}{ccc}
-\sin \left(\theta_{\text {ref } y}(t)\right) & 0 & 1 \\
\cos \left(\theta_{\text {ref } y}(t)\right) \sin \left(\theta_{\text {ref } z}(t)\right) & \cos \left(\theta_{\text {ref } z}(t)\right) & 0 \\
\cos \left(\theta_{\text {ref } y}(t)\right) \cos \left(\theta_{\text {ref } z}(t)\right) & -\sin \left(\theta_{\text {ref } z}(t)\right) & 0
\end{array}\right] \dot{\boldsymbol{\theta}}_{\text {ref }}(t)
$$

So it is possible to determine the reference angular velocity $\boldsymbol{\omega}_{r e f}(t)$ by means of the reference displacement $\boldsymbol{\theta}_{r e f}(t)$ and its derivative $\dot{\boldsymbol{\theta}}_{r e f}(t)$, both available analytically from Eq. (33) as the displacement and speed paths $\theta(t)$ and $\dot{\theta}(t)$ about each axis. 
It is worth noting that the intrinsic singularities of the kinematics written through

Euler angles vanished thanks to the inversion of the equations.

\subsection{Reference tracking}

Once 3-D profiles of position $\left(\overline{\boldsymbol{q}}_{r e f}\right)$ and velocity $\left(\boldsymbol{\omega}_{r e f}\right)$ are obtained either by means of the smoothing of an eigenaxis rotation, Eq. (39) and 40, or through the smoothing of three superimposed 1-D maneuvers, Eq. 41] and (43), it is necessary to develop a control law able to make the spacecraft perform the desired maneuver.

The control law is a quaternion-based control whose task is to minimize the errors between the planned position and planned angular velocity and the current actual ones:

$$
\boldsymbol{u}=\boldsymbol{I}\left(-k_{1} \boldsymbol{q}_{e}-k_{2} \boldsymbol{\omega}_{e}\right)
$$

where $\boldsymbol{q}_{e}$ is the imaginary part of the quaternion error $\overline{\boldsymbol{q}}_{e}$, which expresses the error between the desired reference position at each time instant and the actual one:

$$
\overline{\boldsymbol{q}}_{e}(t)=\overline{\boldsymbol{q}}_{r e f}^{-1}(t) \otimes \overline{\boldsymbol{q}}(t)
$$

${ }_{415}$ and $\boldsymbol{\omega}_{e}$ is the error between the reference velocity at each time instant and the actual one:

$$
\boldsymbol{\omega}_{e}(t)=\boldsymbol{\omega}(t)-\boldsymbol{\omega}_{\text {ref }}(t)
$$

Therefore the control law is designed to make the spacecraft follow the prescribed angular rate and velocity during the maneuver.

It is worth to note that the final control input $\boldsymbol{u}$ may be larger than the maximum torque used to compute the maneuver profiles because of the presence of the disturbing flexibility torque $\boldsymbol{f}$. Therefore, it is necessary to consider a margin between the maximum reference control torque and the actual maximum torque available from actuators.

The process to obtain the control law to perform the smooth maneuver can therefore be summarized as follows:

1. the angular displacement path $\theta(t)$ and angular speed path $\dot{\theta}(t)$ about the Euler axis are computed analytically from Eq. (33) according to the maneuver and spacecraft 
constraints for all the different time phases of the maneuver; they form a reference displacement $\theta_{r e f}(t)$ and a reference speed $\dot{\theta}_{r e f}(t)$ about the eigenaxis, which are converted into a 3 -D reference position $\overline{\boldsymbol{q}}_{r e f}(t)$ and a 3 -D reference velocity $\boldsymbol{\omega}_{r e f}(t)$ through Eq. (39) and 40);

2. alternatively, the angular displacement path and angular speed path can be computed separately about each principal axis and composed into two vectors: $\boldsymbol{\theta}_{\text {ref }}(t)$ and $\dot{\boldsymbol{\theta}}_{r e f}(t)$; the former is then converted into quaternion form $\overline{\boldsymbol{q}}_{r e f}(t)$ through Eq. (39) again, while the latter is converted into a 3 -D reference velocity by means of Eq. 43);

3. the control law is expected to make the spacecraft follow the prescribed position and velocity paths during the maneuver, $\overline{\boldsymbol{q}}_{r e f}(t)$ and $\boldsymbol{\omega}_{r e f}(t)$, so the control law of Eq. (44) is adopted, where the errors are computed with respect to the actual position and velocity at each time instant during the maneuver.

\section{Simulations results}

\subsection{1-D simulation}

A first simulation has been carried out in a 1-D setting in order to show how the trigonometric smoothing technique reduces the excitation of a flexible structure while performing a planar rotation.

The flexible structure is composed by two beams joint together with a hinge, equal to that of Fig. 3. whose mechanical properties are shown in table 1

Table 1: Mechanical properties of the 1-D structure.

\begin{tabular}{lrrlrr}
\hline & Beams & \multicolumn{3}{c}{ Real hinges } \\
Parameter & Beam 1 & Beam 2 & Parameter & Hinge 1 & Hinge 3 \\
\hline$L[\mathrm{~m}]$ & 1 & 1 & $k\left[\mathrm{~N} \mathrm{~m}^{-1}\right]$ & 1200 & 1300 \\
$M[\mathrm{~kg}]$ & 10 & 9 & $c\left[\mathrm{~N} \mathrm{~s} \mathrm{~m}^{-1}\right]$ & 5 & 5 \\
$E I\left[\mathrm{~N} \mathrm{~m}^{2}\right]$ & 1840 & 1850 & $r_{0}[\mathrm{~m}]$ & 1 & \\
$c\left[\mathrm{~N} \mathrm{~s} \mathrm{~m}^{-1}\right]$ & 5 & 5 & & & \\
\hline
\end{tabular}

In this simulation, no disturbance torque affects the motion. The rotation is performed by following both the bang-off-bang acceleration profile and an equivalent profile 
obtained by applying the versine/cosine smoothing technique presented in Sec. 3.1 in order to show how the flexible appendage behaves in the two cases. The the torque produced by the oscillation of the flexible structure, $f$, is chosen as a metrics to represent how the different profiles of acceleration trigger the appendage. The fixed parameters of the rotation are displayed in Table 2.

Table 2: Parameters of the 1-D simulation.

\begin{tabular}{lcc}
\hline Parameter & Symbol & Value \\
\hline Desired position $\left[^{\circ}\right]$ & $\theta_{d}$ & 180 \\
Maximum acceleration $\left[\mathrm{rad} \mathrm{s}^{-1}\right]$ & $\ddot{\theta}_{\max }$ & 0.01 \\
Minimum allowed duration of the rotation $[\mathrm{s}]$ & $t_{f \min }$ & 35.5 \\
Available time for the rotation $[\mathrm{s}]$ & $t_{f}$ & 40 \\
\hline
\end{tabular}

Fig. 9 shows how the original bang-off-bang acceleration profile is converted into an equivalent smoother profile by applying the discussed smoothing process. It can be seen that the main constraints (duration and maximum acceleration) remain unchanged.

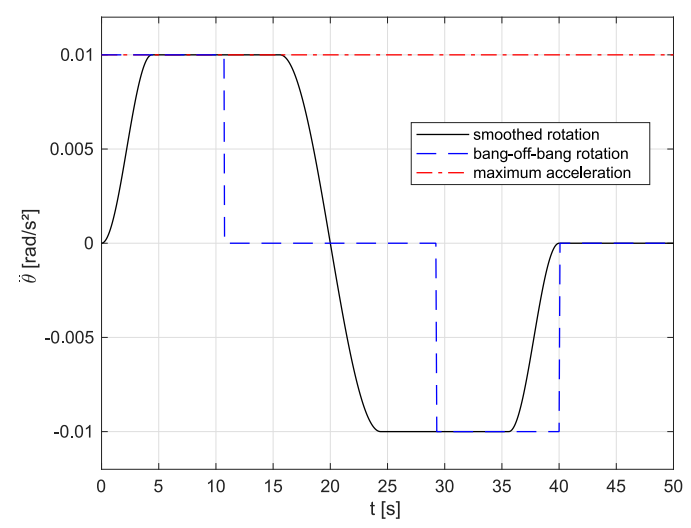

Figure 9: Acceleration profiles of the bang-off-bang and the smoothed rotation.

Fig. 10 shows the flexibility torque due to the oscillation of the structure. It can be seen that the smoothed rotation triggers such oscillation considerably less than the original bang-off-bang rotation.

This simulation shows how a bang-off-bang acceleration profile can be smoothed without changing its constraints. Moreover, it also shows that the application of the smooth- 


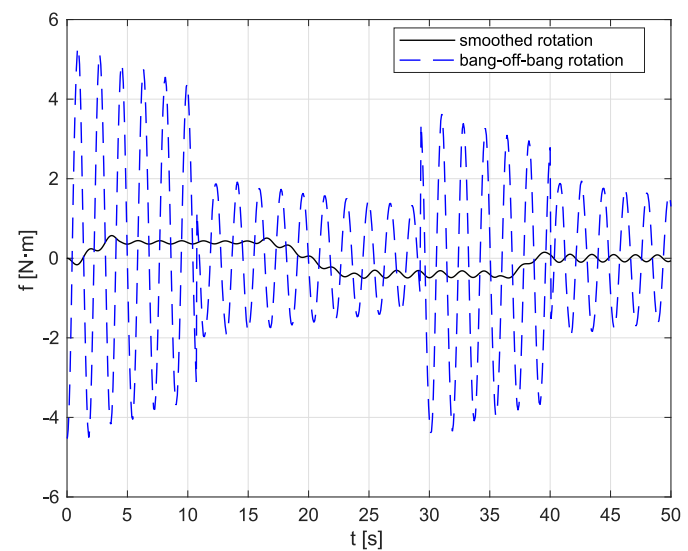

Figure 10: Flexibility torques of the bang-off-bang and the smoothed rotation.

ing technique in planar rotations is capable of reducing the oscillation of the flexible structure.

\subsection{3-D simulations}

Another simulation has been carried out of a generic medium-size satellite performing smoothed 3-D rest-to-rest slew maneuvers. The spacecraft is equipped with a generic flexible slim appendage which is not aligned with any of the main axes of inertia of the vehicle. Both the eigenaxis rotation and the 3-D smoothed maneuver have been simulated in order to analyze the effectiveness of the two techniques. Two test cases have been analyzed: they differ each other in the parameters of the maneuver and the properties of the spacecraft, while the structural properties of the appendage remain unchanged.

The model of the appendage is equal to that of the previous simulation, but now it is able to move in a 3 -D frame. Defining axis $\hat{y}_{A}$ and $\hat{z}_{A}$ as perpendicular to the beams' main axis, such structure has the mechanical properties displayed in Table 3

The position of the flexible structure with respect to the spacecraft main body is given by matrix $\boldsymbol{R}_{B \leftarrow A}$, the following values of the Euler angles being used for the first test case: 
Table 3: Mechanical properties of the 3-D structure.

\begin{tabular}{lrrlrr}
\hline & Beams & \multicolumn{3}{c}{ Real hinges } \\
Parameter & Beam 1 & Beam 2 & Parameter & Hinge 1 & Hinge 3 \\
\hline$L[\mathrm{~m}]$ & 1 & 1 & $k_{y A}\left[\mathrm{~N} \mathrm{~m}^{-1}\right]$ & 1200 & 1300 \\
$M[\mathrm{~kg}]$ & 10 & 9 & $k_{z A}\left[\mathrm{~N} \mathrm{~m}^{-1}\right]$ & 1200 & 1300 \\
$E I_{y A}\left[\mathrm{~N} \mathrm{~m}^{2}\right]$ & 1840 & 1850 & $c_{y A}, c_{z A}\left[\mathrm{~N} \mathrm{~s} \mathrm{~m}^{-1}\right]$ & 5 & 5 \\
$E I_{z A}\left[\mathrm{~N} \mathrm{~m}^{2}\right]$ & 1920 & 1900 & $r_{0}[\mathrm{~m}]$ & 1 & \\
$c_{y A}, c_{z A}\left[\mathrm{~N} \mathrm{~s} \mathrm{~m}^{-1}\right]$ & 5 & 5 & & & \\
$I_{p}\left[\mathrm{~kg} \mathrm{~m}^{2}\right]$ & 0.1 & 0.1 & & & \\
\hline
\end{tabular}

$$
\begin{aligned}
& \alpha=12^{\circ} \\
& \beta=5^{\circ} \\
& \gamma=35^{\circ}
\end{aligned}
$$

The controller is designed according to Eq. (44) where the gains have been set as $k_{1}=1 \mathrm{~s}^{-2}$ and $k_{2}=2 \mathrm{~s}^{-1}$, which were found through a trial-and-error iterative process and showed to be effective for controlling this system.

The fixed parameters of the maneuver and the spacecraft for the first test case are shown in Table 4

Table 4: Parameters of the 3-D simulation (test case 1).

\begin{tabular}{lcccc}
\hline Parameter & Symbol & \multicolumn{3}{c}{ Value } \\
\hline Desired position $\left[^{\circ}\right]$ & $\boldsymbol{\theta}_{d}$ & {$\left[\begin{array}{lll}135 & 180 & -144\end{array}\right]$} \\
Maximum control torque $[\mathrm{N} \mathrm{m}]$ & $\boldsymbol{u}_{\max }$ & {$\left[\begin{array}{lll}1.95 & 1.55 & 1.75\end{array}\right]$} \\
Moment of inertia of the main body $\left[\mathrm{kg} \mathrm{m}^{2}\right]$ & $\boldsymbol{I}$ & {$\left[\begin{array}{lll}90 & 85 & 100\end{array}\right]$} \\
Minimum allowed duration about each axis $[\mathrm{s}]$ & $\boldsymbol{t}_{f \text { min }}$ & {$\left[\begin{array}{ccc}24.1 & 30.2 & 27.7\end{array}\right]$} \\
Available time for the maneuver $[\mathrm{s}]$ & $t_{f}$ & 45 \\
\hline
\end{tabular}

The actual maximum torque used for computing the acceleration profiles has been set as $75 \%$ of the nominal one, in order to grant a margin for compensating the disturbing 
flexibility torque $\boldsymbol{f}$.

In Fig. 11 the attitude error of the overall maneuver, i.e. the quaternion error referred to the desired final position, is visible. The two smoothing techniques are applied and in both cases the spacecraft is able to reach the desired position with accuracy.

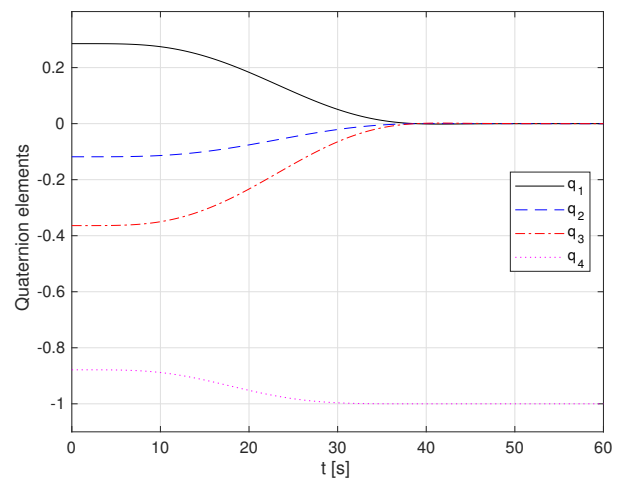

(a) Eigenaxis rotation.

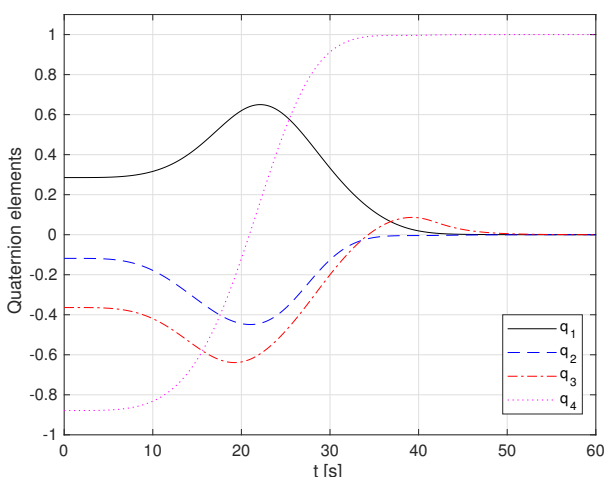

(b) 3-D smoothed maneuver.

Figure 11: Attitude errors (test case 1).

Fig. 12 displays the angular velocities during the eigenaxis rotation and the 3-D smoothed maneuver compared with the planned expected angular velocity. It can be seen that the maximum value in the 3-D smoothed maneuver is larger than expected about the $\hat{\boldsymbol{y}}$ and $\hat{\boldsymbol{z}}$ axes because of the influence of the disturbing torque due to the oscillation of the flexible appendage. On the other hand, the angular velocity during the eigenaxis rotation is smaller, thus reducing the centrifugal forces acting on the structure during the maneuver.

Fig. 13 shows the accelerations for the eigenaxis rotation and the 3-D smoothed maneuver (planned and actual). The first is far lower than the latter on each axis; moreover it is also smoother, so a lower excitation of the flexible structure may be expected. Concerning the 3-D smoothed maneuver, it is possible to see some notable divergences between the planned acceleration and the real one, especially in the dynamics about the $\hat{\boldsymbol{y}}$ axis, because of the oscillation of the flexible appendage.

Fig. 14 shows the control torques needed to perform both the eigenaxis rotation and the 3-D smoothed maneuver and their saturation limits. It is possible to see that while 505 the control torques $\boldsymbol{u}$ in the eigenaxis rotation are always far from the limits, those of 


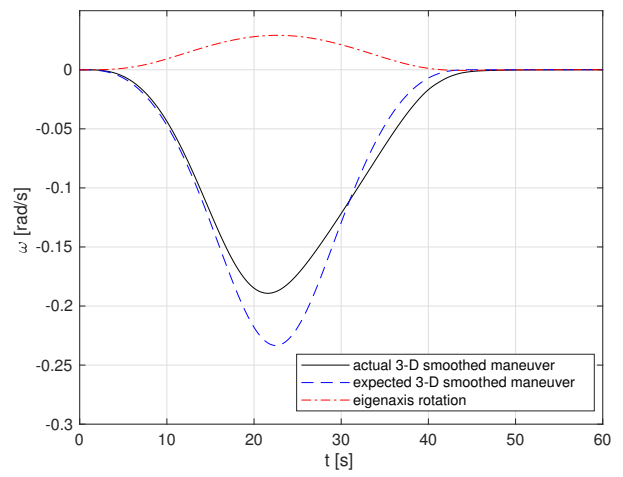

(a) About $\hat{\boldsymbol{x}}$.

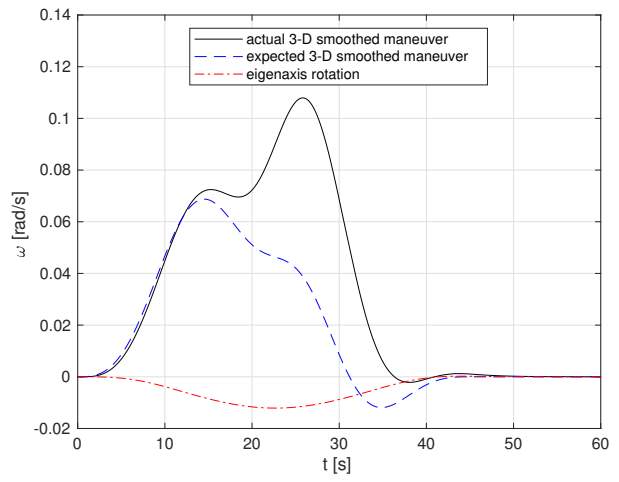

(b) About $\hat{\boldsymbol{y}}$.

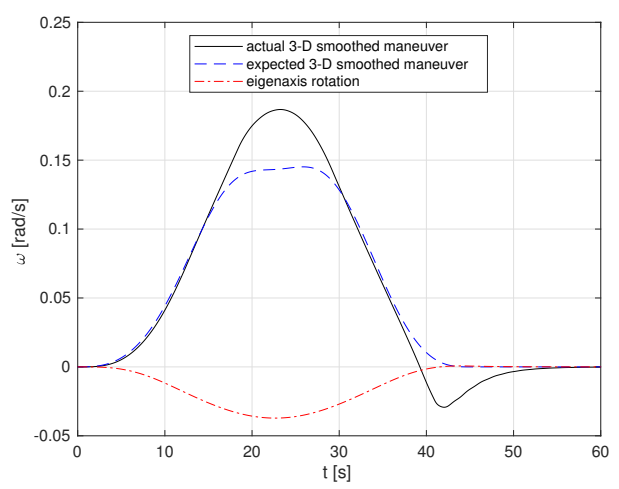

(c) About $\hat{\boldsymbol{z}}$.

Figure 12: Angular velocities (test case 1).

the 3-D smoothed maneuver sometimes reach the upper and lower bounds, especially the one about $\hat{\boldsymbol{z}}$. This happens in spite of the reduction of the maximum torque available for 


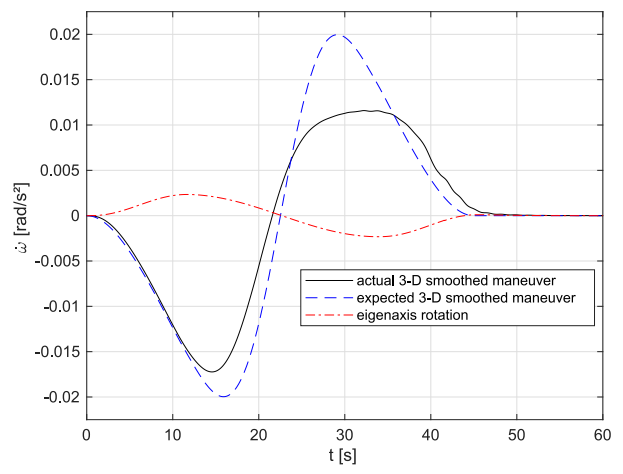

(a) About $\hat{\boldsymbol{x}}$.

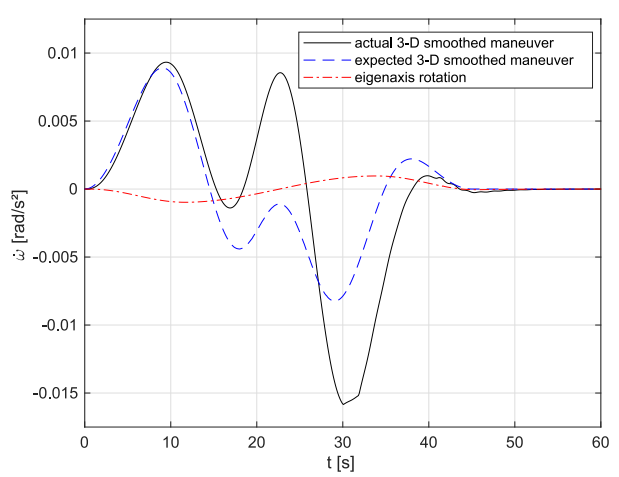

(b) About $\hat{\boldsymbol{y}}$.

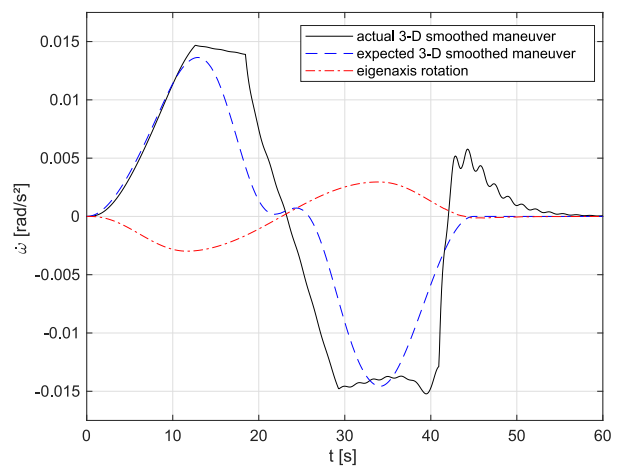

(c) About $\hat{\boldsymbol{z}}$.

Figure 13: Angular accelerations (test case 1).

maneuver planning to $75 \%$ of the nominal maximum one that can be provided by the actuators. However, as it can be seen in the other plots, this does not affect significantly the capability of the spacecraft to perform correctly the maneuver. 


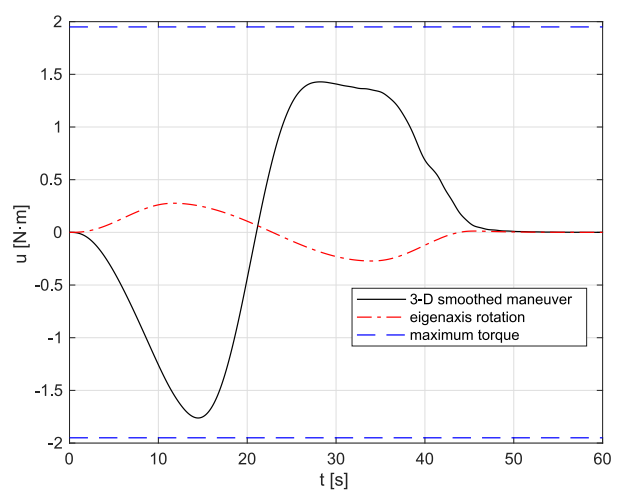

(a) About $\hat{\boldsymbol{x}}$.

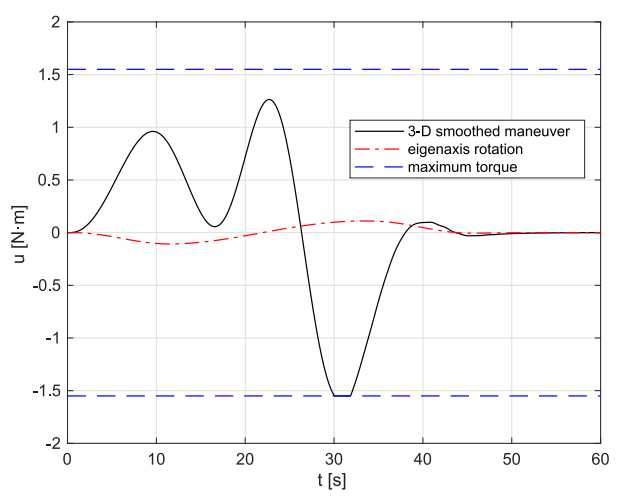

(b) About $\hat{\boldsymbol{y}}$.

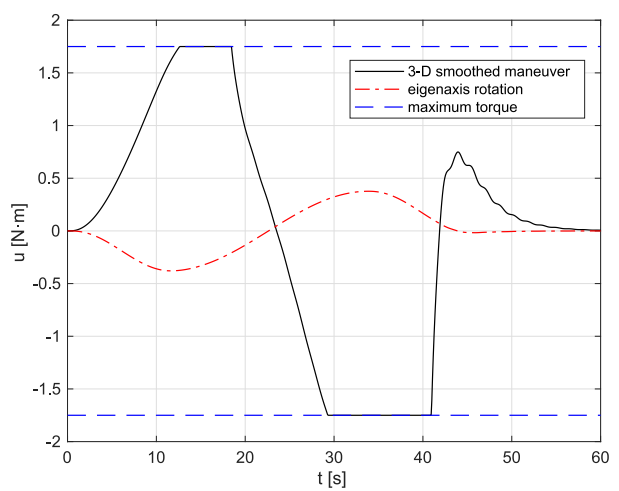

(c) About $\hat{\boldsymbol{z}}$.

Figure 14: Control torques (test case 1).

The following two plots show the outcomes of a comparison between the smoothed maneuvers and a classical proportional-derivative control, in order to investigate how 
the smoothing process improves the performance of the maneuver. Such classical control law is formulated as $\boldsymbol{u}=\boldsymbol{I}\left(-k_{1} \boldsymbol{q}_{e}-k_{2} \boldsymbol{\omega}\right)$, which differs from Eq. (44) as the quaternion error is defined with respect to the final desired position of the maneuver and the velocity error is replaced by the velocity itself. The gains remain unchanged.

Fig. 15 shows the magnitude of the flexibility torque in the three cases and allows to see that both the smooth maneuvers produce a generally lower excitation of the appendage; in particular, the eigenaxis rotation triggers a lighter excitation than the 3-D smoothed maneuver. Moreover, both smooth motions are able to dampen the oscillation to zero in less time.

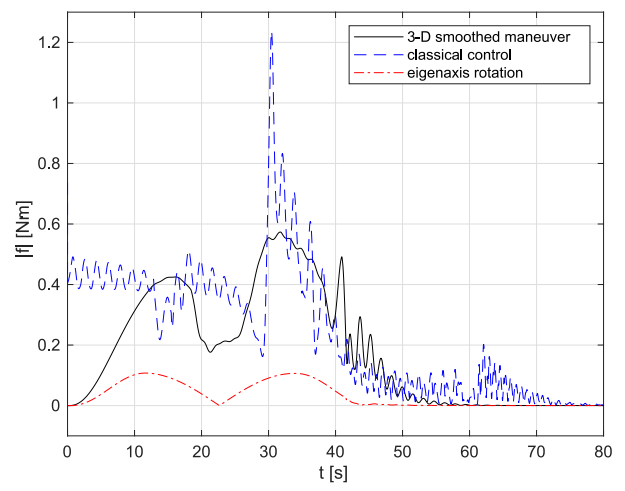

Figure 15: Magnitude of the flexibility torques (test case 1).

In Fig. 16 the overall cost of the different maneuvers are compared. The plot shows the total angular impulse, which is defined as

$$
\Lambda=\int_{0}^{t_{f}}\|\boldsymbol{u}\| d t
$$

and provides a good measurement of the total cost of the maneuver. It can be seen that the cost of the eigenaxis rotation in terms of total angular impulse is remarkably smaller than that of the 3-D smoothed maneuver, which in turn is sensibly lower than that of the classical control.

From the plots it is possible to note that in general the dynamics about the $\hat{z}$ axis in the 3-D smoothed maneuver presents more sharpness than the two others. In particular, 530 it can be seen in Fig. $14 \mathrm{c}$ that the torque which is needed to properly perform the maneuver and compensate the disturbance would exceed the maximum available torque 


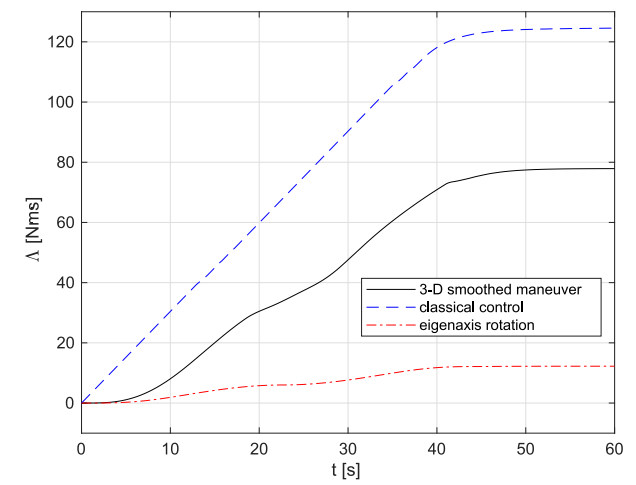

Figure 16: Total angular impulses (test case 1).

for large parts of the maneuver and is therefore limited to $\boldsymbol{u}_{\max }$. By comparing that plot with that of Fig. 13c it is possible to see that a sudden variation of the control torque and of the acceleration happens after the end of the saturation of the control torque. This allows to hypothesize that the motion could be made smoother by increasing the torque margin which is used to compensate the disturbance torque, which is now at $25 \%$ of the nominal maximum torque. However, it must also be taken into account that a reduction of the maximum reference control torque in the maneuver planning would reduce the possible maneuvers that can be performed through the smoothing process.

The second 3-D test case uses the same appendage whose mechanical properties are displayed in Table 3, but now it is located on a different position in BRF:

$$
\begin{aligned}
& \alpha=140^{\circ} \\
& \beta=75^{\circ} \\
& \gamma=350^{\circ}
\end{aligned}
$$

while the fixed parameters of the maneuver and the spacecraft are now those shown in Table 5. In this test case, the maximum control torque has been increased in order to get a faster rotation and see how the controller is able to cope with increased oscillations.

545 Again, the actual maximum torque used for computing the acceleration profiles is $75 \%$ of the nominal one.

Fig. 17 shows the attitude error of the overall maneuver for the two smoothing tech- 
Table 5: Parameters of the 3-D simulation (test case 2).

\begin{tabular}{lccc}
\hline Parameter & Symbol & \multicolumn{2}{c}{ Value } \\
\hline Desired position $\left[^{\circ}\right]$ & $\boldsymbol{\theta}_{d}$ & {$\left[\begin{array}{ccc}-153 & 162 & 108\end{array}\right]$} \\
Maximum control torque $[\mathrm{N} \mathrm{m}]$ & $\boldsymbol{u}_{\max }$ & {$\left[\begin{array}{ccc}5 & 5.5 & 4.5\end{array}\right]$} \\
Moment of inertia of the main body $\left[\mathrm{kg} \mathrm{m}^{2}\right]$ & $\boldsymbol{I}$ & {$\left[\begin{array}{ccc}180 & 200 & 205\end{array}\right]$} \\
Minimum allowed duration about each axis $[\mathrm{s}]$ & $\boldsymbol{t}_{f \min }$ & {$\left[\begin{array}{ccc}19.7 & 20.8 & 18.6\end{array}\right]$} \\
Available time for the maneuver $[\mathrm{s}]$ & $t_{f}$ & 40 \\
\hline
\end{tabular}

niques. Again, the spacecraft reaches the desired attitude correctly.

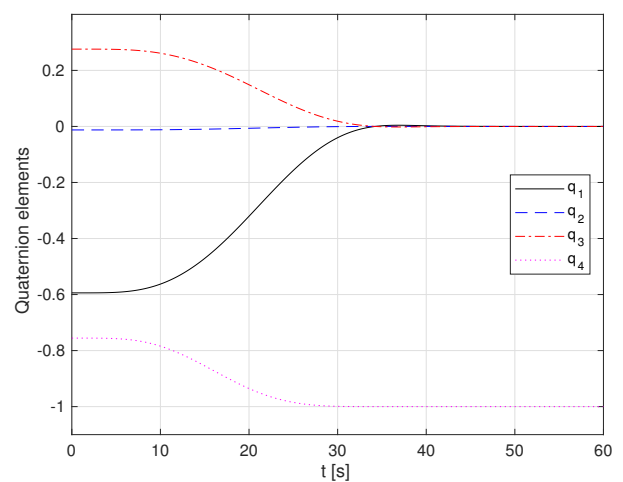

(a) Eigenaxis rotation.

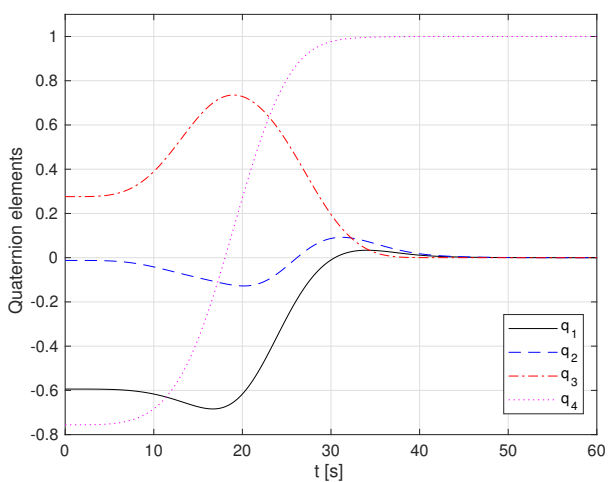

(b) 3-D smoothed maneuver.

Figure 17: Attitude errors of the smoothed maneuvers (test case 2).

In Fig. 18 the accelerations about each axis are compared once more. As in the 3-D smoothed maneuver (both the expected and the actual ones).

Figs. 19 and 20 show that the smoothed maneuvers are better than a classical control in reducing the excitation of the flexible appendage and the total cost of the maneuver. In particular, it can be seen that the increase of the velocity of the maneuver leads to a strong oscillation when the classical control is applied, while the smoothing techniques are able to mitigate it.

The test case shows that the smoothed techniques are preferable to the classical control also in the case of fast maneuvers. Indeed, even if the classical control is able 


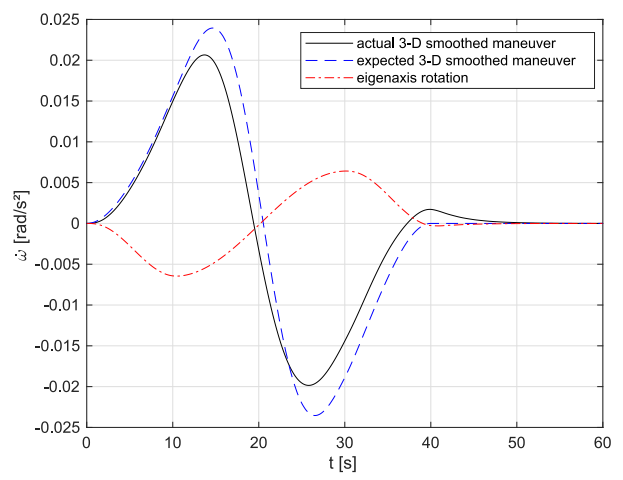

(a) About $\hat{\boldsymbol{x}}$.

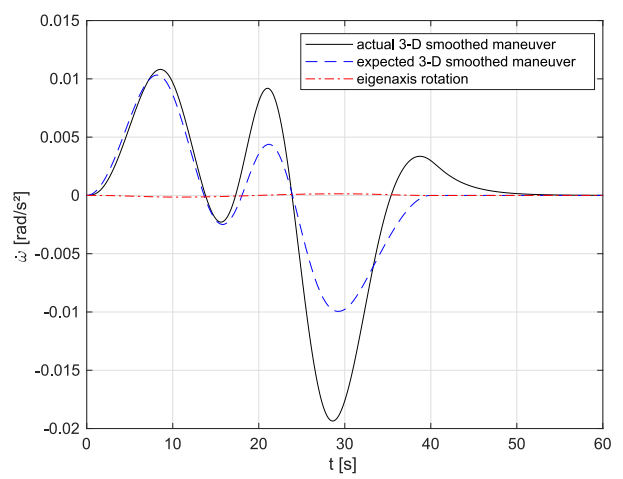

(b) About $\hat{\boldsymbol{y}}$.

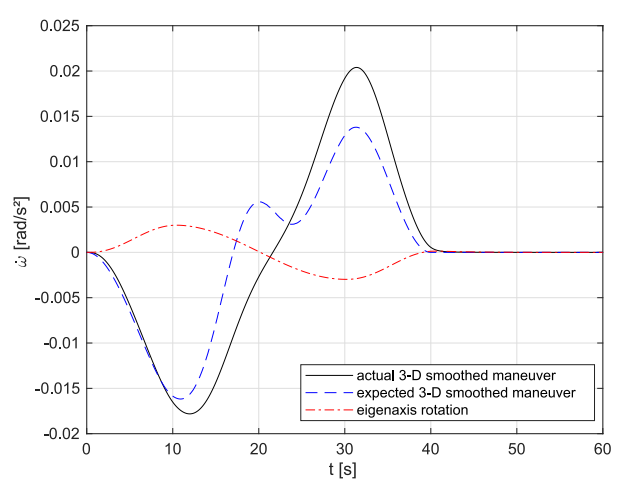

(c) About $\hat{\boldsymbol{z}}$.

Figure 18: Angular accelerations (test case 2).

to complete the rotation in a smaller amount of time, its larger velocity produces a

stronger residual oscillation of the flexible appendage, which lasts far beyond the end of 


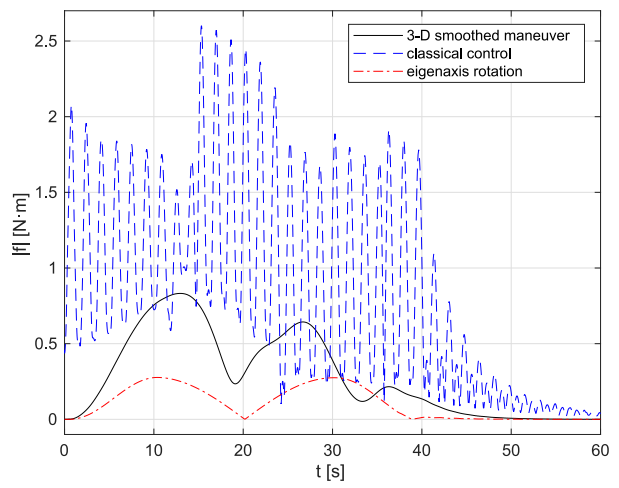

Figure 19: Magnitude of the flexibility torques (test case 2).

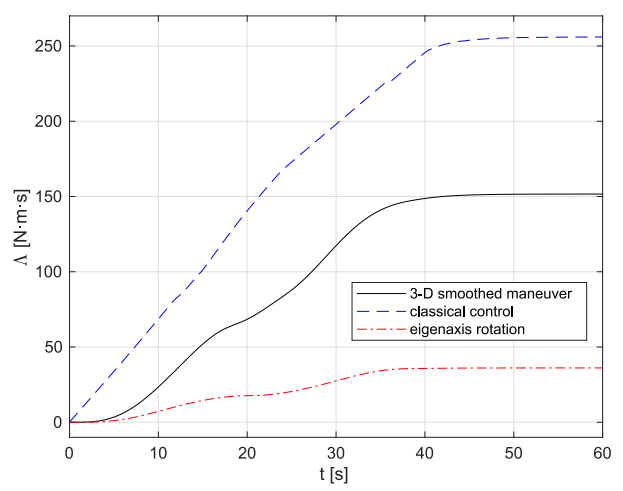

Figure 20: Total angular impulses (test case 2).

the smoothed maneuvers.

\section{Conclusion}

An approach to design analytically-defined rest-to-rest reorientation maneuvers with low jerk was developed. It was tested in simulation with a multi-body structural model based on the lumped-parameter method. Such an approach allows the control engineer to prescribe some parameters of the maneuver (desired position, maximum duration) and the spacecraft (moments of inertia, maximum control torques) and design a 1-D low-jerk rotation under the imposed constraints.

Two methods to extend this technique to $3-\mathrm{D}$ maneuvers were presented. The first 570 is based on the smoothing of a 1-D rotation about the Euler axis, so as to obtain a 
global 3-D maneuver that can use the 1-D smoothing procedure on the eigenangle. The second method is more general: the 1-D analytical approach was applied to each rotation axis separately, obtaining three profiles of angular position that are used to find the corresponding profiles of velocity by means of inverse kinematics.

Both presented smoothing techniques are very efficient from a computational point of view: they make use of algebraic computations only and no calculus is required. The computation of the angular velocity is not computationally expensive either, as the profiles are computed by means of closed-form analytical equations and then simply converted into a 3-D motion.

The two methods were simulated and both of them shown to be effective. The rotation about the Euler axis is in general better than the generic 3-D smoothed maneuver in reducing the excitation of the flexible structure and the total cost of the maneuver. Since it is also simpler to implement, the smoothing of a bang-bang Euler axis rotation provides a useful trajectory for 3-axis rotations of spacecraft with multi-body or flexible appendages.

\section{References}

[1] C. Adams, W. Singhose, Robust constant-amplitude input shapers with selectable duration, J. Guid. Control Dyn. 41 (12) (2018) 2543-2553. doi:10.2514/1.G003720

[2] R. Nadafi, M. Kabganian, A. Kamali, M. Hossein Nejad, Super-twisting sliding mode control design based on lyapunov criteria for attitude tracking control and vibration suppression of a flexible spacecraft, Meas. Control 52 (7-8) (2019) 814-831. doi:10.1177/0020294019847696

[3] C. Zhong, Z. Chen, Y. Guo, Attitude control for flexible spacecraft with disturbance rejection, IEEE Trans. Aerosp. Electron. Syst. 53 (1) (2017) 101-110. doi:10.1109/TAES.2017.2649259

[4] R. Yan, Z. Wu, Attitude stabilization of flexible spacecrafts via extended disturbance observer based controller, Acta Astronaut. 133 (2017) 73-80. doi:10.1016/j.actaastro.2017.01.004

[5] B. A. Albassam, Attitude and vibration control system modeling and simulation for a spacecraft using smart materials, in: Proceedings of the Third International Conference on Advances in Mechanical and Automation Engineering - MAE 2015, Rome, Italy, 2015. doi:10.15224/ 978-1-63248-080-4-71.

600 [6] L. Mazzini, Flexible Spacecraft Dynamics, Control and Guidance, Springer, Switzerland, 2016. doi : 10.1007/978-3-319-25540-8

[7] P. Gili, M. Battipede, L. Massotti, Modelling and neural control of satellites with flexible appendages, in: Proceedings of the 6th Conference on Dynamics and Control of Systems and Structures in Space, Riomaggiore, Italy, 2004. 
[8] N. Hogan, Adaptive control of mechanical impedance by coactivation of antagonist muscles, IEEE Trans. Automat. Contr. 29 (8) (1984) 681-690. doi:10.1109/TAC.1984.1103644

[9] M. J. E. Richardson, T. Flash, Comparing smooth arm movements with the two-thirds power [1 law and the related segmented-control hypotheses, J. Neurosci. 22 (18) (2002) 8201-8211. doi: 10.1523/JNEUROSCI. 22-18-08201.2002

610 [10] K. D. Bilimoria, B. Wie, Time-optimal three-axis reorientation of a rigid spacecraft, J. Guid. Control Dyn. 16 (3) (1993) 446-452. doi:10.2514/3.21030

[11] J.-J. Kim, B. N. Agrawal, Experiments on jerk-limited slew maneuvers of a flexible spacecraft, in: AIAA Guidance, Navigation, and Control Conference and Exhibit, Keystone, CO, USA, 2006. doi:10.2514/6.2006-6187

[12] M. J. Bell, J. L. Junkins, Near minimum-time three dimensional maneuvers of rigid and flexible spacecraft, J. Astronaut. Sci. 42 (4) (1994) 421-438.

[13] J.-J. Kim, B. N. Agrawal, Rest-to-rest slew maneuver of three-axis rotational flexible spacecraft, IFAC Proceedings Volumes 41 (2) (2008) 12054-12060. doi:10.3182/20080706-5-KR-1001.02040

[14] A. Caubet, J. D. Biggs, A motion planning method for spacecraft attitude maneuvers using single polynomials, in: Proceedings of the AAS/AIAA Astrodynamics Specialist Conference 2015, Vail, CO, USA, 2015.

[15] D. Lee, Y.-J. Song, Single-axis, spin-to-spin slew maneuvers under a finite jerk constraint, J. Guid. Control Dyn. 42 (9) (2019) 2116-2123. doi:10.2514/1.G004351

[16] Y. Hu, B. Wu, Y. Geng, Y. Wu, Smooth time-optimal attitude control of spacecraft, Proc. Inst. Mech. Eng. G 233 (7) (2018) 2331-2343. doi:10.1177/0954410018776531

[17] F. Schiavo, L. Viganò, G. Ferretti, Object-oriented modelling of flexible beams, Multibody Syst. Dyn. 15 (3) (2006) 263-286. doi:10.1007/s11044-006-9012-8

[18] A. K. Banerjee, Flexible Multibody Dynamics: Efficient Formulations and Applications, John Wiley \& Sons, 2016. doi:10.1002/9781119015635

630 [19] A. Di Meglio, A. Ercoli Finzi, Minimum time control of flexible spacecraft by hamilton's principle, Meccanica 32 (6) (1997) 555-565. doi:10.1023/A:1004204510722

[20] F. Schiavo, M. Lovera, A. Astolfi, Magnetic attitude control of spacecraft with flexible appendages, in: Proceedings of the 45th IEEE Conference on Decision and Control, IEEE, San Diego, CA, USA, 2006. doi:10.1109/CDC.2006.377766.

635 [21] S. Shahriari, S. Azadi, M. M. Moghaddam, An accurate and simple model for flexible satellites

1] for three-dimensional studies, J. Mech. Sci. Technol. 24 (6) (2010) 1319-1327. doi:10.1007/ s12206-010-0329-0

[22] L. Meirovitch, Fundamentals of Vibrations, McGraw-Hill, 2001.

[23] A. W. Leissa, M. S. Qatu, Vibrations of Continuous Systems, McGraw-Hill, 2011.

640 [24] K.-H. Chang, e-Design, Academic Press, 2015. doi:10.1016/C2009-0-63076-2.

[25] J. D. Biggs, L. Colley, Geometric attitude motion planning for spacecraft with pointing and actuator constraints, J. Guid. Control Dyn. 39 (7) (2016) 1672-1677. doi:10.2514/1.G001514

[26] B. Wie, Space Vehicle Dynamics and Control, 2nd Edition, American Institute of Aeronautics and 
Astronautics, 2008. doi:10.2514/4.860119. 